\title{
Design of Spacecraft Formation Orbits Relative to a Stabilized Trajectory
}

\author{
F. Y. Hsiao* and D. J. Scheeres ${ }^{\dagger}$ \\ University of Michigan, Ann Arbor, Michigan 48109-2140
}

\begin{abstract}
The design of spacecraft formation orbits traveling relative to an unstable trajectory is investigated. We implement the designs with position and velocity feedback control laws, so that the entire constellation is forced to follow a similar path relative to a nominal trajectory. We find sufficient conditions under which the relative motion of the spacecraft constellation is stable and a controller can be designed. We consider the problem of specifying the orientation of the formation orbit, which is equivalent to eigenvector placement, and show how these modes can be combined to force a formation to fly in a range of orientations. Applications of our approach to relative motion in rotating and nonrotating systems are given.
\end{abstract}

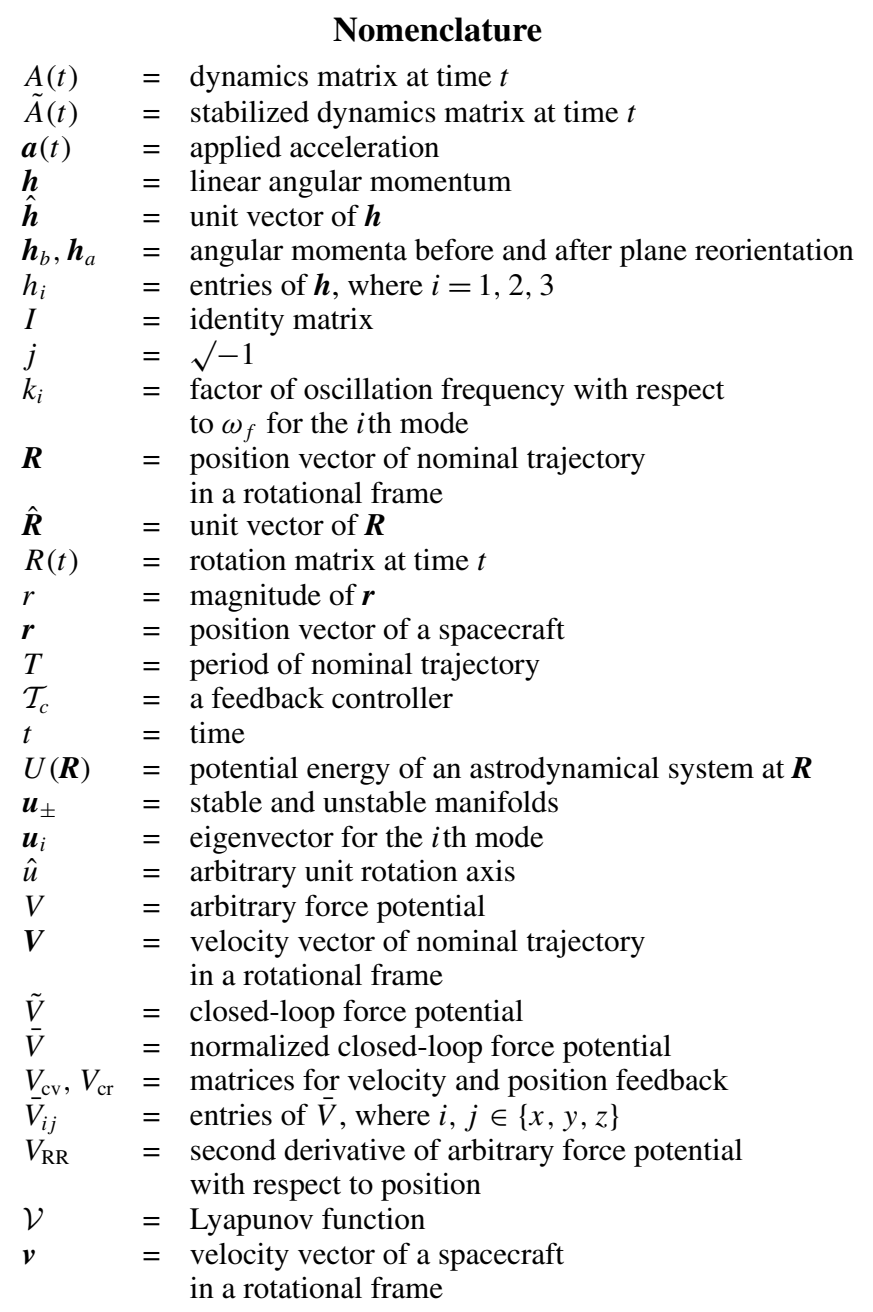

Received 19 February 2004; revision received 7 August 2004; accepted for publication 7 August 2004. Copyright (C) 2004 by F. Y. Hsiao and D. J. Scheeres. Published by the American Institute of Aeronautics and Astronautics, Inc., with permission. Copies of this paper may be made for personal or internal use, on condition that the copier pay the $\$ 10.00$ per-copy fee to the Copyright Clearance Center, Inc., 222 Rosewood Drive, Danvers, MA 01923; include the code 0731-5090/05 \$10.00 in correspondence with the CCC.

${ }^{*} \mathrm{Ph} . \mathrm{D}$. Candidate, Department of Aerospace Engineering; currently Assistant Professor, Department of Aerospace Engineering, Tamkang University, Tamsui 251, Taiwan, Republic of China; fyhsiao@qmail.com.

${ }^{\dagger}$ Associate Professor, Department of Aerospace Engineering; scheeres@ umich.edu. Associate Fellow AIAA.

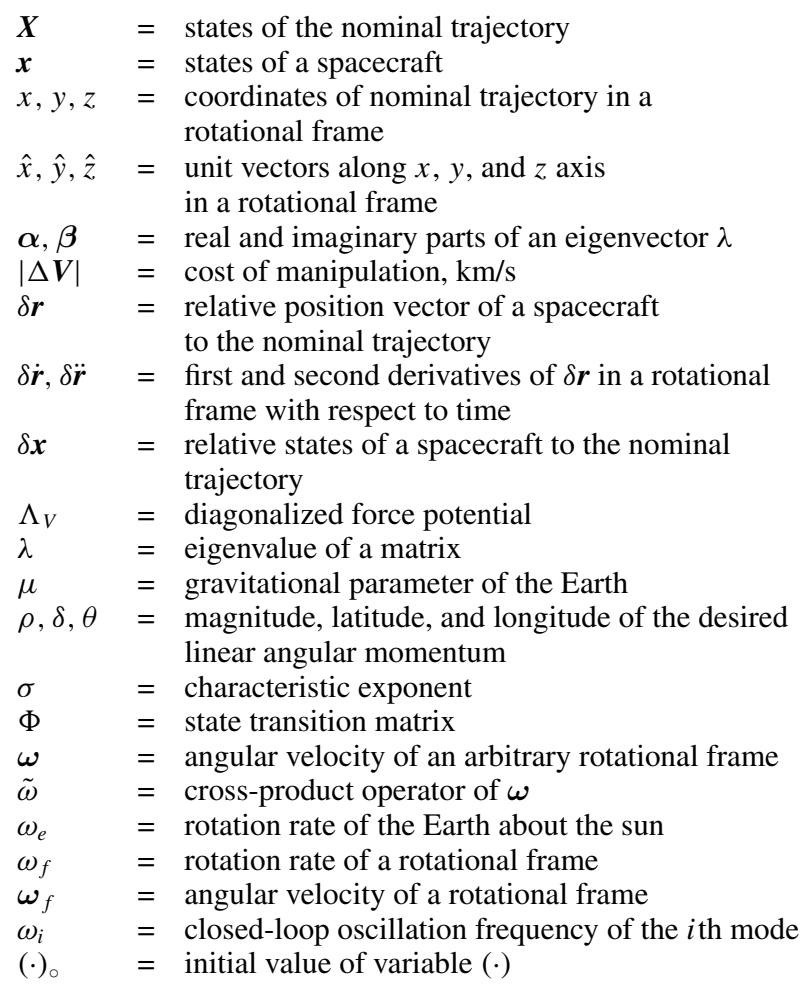

\section{Introduction}

AENERAL control law to stabilize the relative motion about an $A$ unstable trajectory in a Hamiltonian system and related methods of relative trajectory design are studied in this paper. Among the possible applications of this work is formation flight about a halo orbit in the Hill or a restricted three-body problem. A halo orbit is an unstable periodic orbit in the vicinity of libration points in the sunEarth system. This work is motivated by potential future applications of spacecraft formation flight for interferometric imaging of distant stellar systems. Flying formations of spacecraft in the vicinity of $L_{2}$ halo orbits has been recognized as a feasible environment for this application. Results of this study will contribute to the possible application of formation-flight techniques in this unstable environment.

The idea for using the center manifold of a halo orbit for formation flight was proposed in Ref. 1 and generalized in Ref. 2. In this paper we seek to develop a general design methodology, including general control law and trajectory design, for the specification of relative trajectories about an arbitrary orbit. For definiteness we concentrate on motion about a periodic halo orbit. Our current approach to the design and control of formation-flight trajectories should be 
distinguished from previous work, such as that reported in Refs. 3 and 4 , as those efforts focus on the control and design of formation flight in near-Earth orbits. The current work in this paper is an extension of the work reported in Ref. 5, which investigated the feasibility of the local-time algorithm for the description of relative motion about a halo orbit and reported in Ref. 2, where the concept of a stabilizing control was introduced. Here we investigate the general rule from which different controllers are designed to stabilize the relative motion and to reshape the relative trajectory.

This work is developed for application to interferometric imaging using distributed spacecraft formations. The goal of this analysis is to develop a general control law that allows formation-flight dynamics to be assigned so that the relative motion between spacecraft in the formation cover the image plane and reconstruct an image. Our approach will be used in conjunction with innovative interferometric imaging approaches that can relieve the tight relative position control constraints with which previous system concepts have had to deal. ${ }^{6}$ Thus, by defining the preferred planes of motion for our formation orbit we control the preferred imaging surfaces as a function of time. In Ref. 6 it is shown that simple circular relative motions in a formation can successfully satisfy the interferometric imaging requirements; thus, we focus on the design of a circular orbit relative to an unstable halo periodic orbit.

In this paper we find a condition under which the relative motion about a general trajectory is stabilized. Then we apply our algorithm to relative motion about a halo orbit in the Hill three-body problem. The control law creates a linear, time-varying system about the periodic orbit, where all motion winds on tori about the periodic orbit. It can be shown that these orbits are stable over both short and long periods of time and that their winding number about the periodic orbit can be controlled by the gain of the feedback law. Given a desired relative orbit plane and shape, we develop several algorithms to implement the necessary controller, and, by evaluating the $\Delta \boldsymbol{V}$ cost of these controllers over a halo orbit period, show the feasibility of implementing these controls.

Specifically, we find that a negative definite force potential matrix can generate stable motions relative to a nominal halo orbit. With this result, we develop several approaches for trajectory design. We first investigate position-only feedback; however, caused by the presence of a Coriolis force, we only find relative circular trajectories that exist on the ecliptic plane, which restrict the interferometric imaging application. To find out-of-plane motion for a position-only feedback control we must relax the circular trajectory constraint and instead obtain near-circular trajectories. ${ }^{7}$ We next investigate position-and-velocity feedback controls that allow us to generate a simple three-dimensional harmonic oscillation about the trajectory, which gives us more freedom in design. Based on the same approach, we develop a control law that enables us to perform relative trajectory plane reorientation and to design formations with fixed relative trajectory orientations in an inertial frame. Finally, we evaluate the $\Delta \boldsymbol{V}$ costs of implementing these controllers and show that the required accelerations are small enough to be implemented by a low-thrust engine.

\section{Model of Spacecraft Motion}

\section{General Equations of Motion in a Rotational Frame}

To model the formation flight of spacecraft, we consider the formation as a constellation of spacecraft flying in the vicinity of a nominal trajectory. The nominal trajectory is defined as the solution to the nonlinear equations of motion in a rotational frame. The dynamics of the spacecraft formation will, in turn, be defined as the solution to the linearized motion about the nominal trajectory. We now derive the relevant equations of motion for these systems.

Assume the triad $(x, y, z)$ is the coordinates of the nominal trajectory in a frame rotating about the $\hat{z}$ axis with a constant rotation rate $\omega_{f}$. If the force potential is defined as $U(\boldsymbol{R})$, we can write the equations of motion in the rotating frame as

$$
\ddot{\boldsymbol{R}}+2 \boldsymbol{\omega}_{f} \times \dot{\boldsymbol{R}}+\boldsymbol{\omega}_{f} \times\left(\boldsymbol{\omega}_{f} \times \boldsymbol{R}\right)=\frac{\partial U(\boldsymbol{R})}{\partial \boldsymbol{R}}
$$

where $\boldsymbol{R}=\sqrt{ }\left(x^{2}+y^{2}+z^{2}\right) \hat{\boldsymbol{R}}$ is the position vector of the spacecraft. In terms of coordinates, Eq. (1) can be expressed as

$$
\begin{gathered}
\ddot{x}-2 \omega_{f} \dot{y}=\frac{\partial V}{\partial x} \\
\ddot{y}+2 \omega_{f} \dot{x}=\frac{\partial V}{\partial y} \\
\ddot{z}=\frac{\partial V}{\partial z} \\
V=U(\boldsymbol{R})+\frac{1}{2} \omega_{f}^{2}\left(x^{2}+y^{2}\right)
\end{gathered}
$$

We can view $\omega_{f}$ as a free parameter and $V$ as an arbitrary force potential so that all space missions satisfying these equations of motion can be analyzed. If $\omega_{f}$ is taken to be zero, the preceding equations of motion degenerate to those in an inertial frame.

\section{Hill Problem}

A spacecraft's trajectory in the vicinity of a halo orbit in the sunEarth system is highly unstable and non-Keplerian. To compute the nonlinear equations of motion, we use an approximation to the restricted three-body problem, known as the Hill problem. ${ }^{8}$ The three-dimensional motion is governed by equations of motion with the same structure as Eqs. (2-4), where the frame is centered at the Earth, $x$ axis points toward the Earth from the sun, the $z$ axis points normal to the Earth orbital plane, and the $y$ axis completes the triad. In this problem, $\omega_{e}$ is the rotation rate of the Earth's orbit about the sun, and the force potential is taken as

$$
V=\mu / r+\frac{1}{2} \omega_{e}^{2}\left(3 x^{2}-z^{2}\right)
$$

There exist two equilibrium points with coordinates $\left[ \pm\left(\mu / 3 \omega_{e}^{2}\right)^{1 / 3}\right.$, $0,0]$ (Ref. 8) in this system.

The Hill equations have been numerically integrated with proper initial conditions to find a periodic halo orbit with a period of 179 days, similar to the Genesis halo orbit during its main mission phase. ${ }^{9}$ This orbit has two pairs of stable oscillation modes, one with the same period as that of the rotating frame leading to unity eigenvalues, with the other slightly longer leading to a pair of eigenvalues on the unit circle, and one pair of characteristic exponents $\sigma= \pm 4.757 \times 10^{-7} / \mathrm{s}$ (a characteristic time of 24.3 days), one of which causes a hyperbolic instability. The choice of this orbit is somewhat arbitrary, as the methods we are developing can be applied to a much wider range of nominal orbits. ${ }^{2}$

\section{Linearized Dynamics}

To derive equations for relative motion between the spacecraft, we assume the spacecraft are on a nonperiodic orbit in the vicinity of the nominal trajectory. Assume the nominal trajectory (which can correspond to a spacecraft) has a trajectory defined as $\boldsymbol{R}\left(t ; \boldsymbol{R}_{\circ}, \boldsymbol{V}_{\circ}\right)$ with the property $\boldsymbol{R}(t+T)=\boldsymbol{R}(t)$. Naturally, the velocity, $\boldsymbol{V}\left(t ; \boldsymbol{R}_{\circ}, \boldsymbol{V}_{\circ}\right)$, is also periodic. Similarly, we can define the position and velocity vectors for a neighboring spacecraft by $\boldsymbol{r}\left(t ; \boldsymbol{r}_{\circ}, \boldsymbol{v}_{\circ}\right)$ and $\boldsymbol{v}\left(t ; \boldsymbol{r}_{\circ}, \boldsymbol{v}_{\circ}\right)$. Because the second spacecraft is initially in the vicinity of the first spacecraft, linear systems theory can be applied to describe their relative dynamics. We define $\boldsymbol{X}=[\boldsymbol{R} ; \boldsymbol{V}], \boldsymbol{x}=[\boldsymbol{r} ; \boldsymbol{v}]$, and $\delta \boldsymbol{x}$ as the difference between them, then the dynamics of $\delta \boldsymbol{x}$ can be approximated by ${ }^{5}$

$$
\begin{gathered}
\delta \boldsymbol{x}=\boldsymbol{x}-\boldsymbol{X} \\
\delta \dot{\boldsymbol{x}}=A(t) \delta \boldsymbol{x} \\
\delta \boldsymbol{x}=\Phi\left(t, t_{\circ}\right) \delta \boldsymbol{x}_{\circ} \\
A(t)=\left[\begin{array}{c|c}
0 & I_{3 \times 3} \\
\hline V_{\mathrm{RR}} & 2 \omega_{f} J
\end{array}\right]
\end{gathered}
$$




$$
\begin{gathered}
J=\left[\begin{array}{ccc}
0 & 1 & 0 \\
-1 & 0 & 0 \\
0 & 0 & 0
\end{array}\right] \\
\Phi\left(t_{\circ}, t_{\circ}\right)=I_{6 \times 6}
\end{gathered}
$$

where $A(t)$ is periodic, $\Phi$ is the state transition matrix, and $\delta \boldsymbol{x}_{\circ}$ is the initial offset from the first spacecraft, that is, the nominal periodic orbit. Any other spacecraft in the vicinity of the nominal trajectory will follow the same dynamics, albeit with different initial conditions $\delta \boldsymbol{x}_{\circ}$. Thus, the dynamics of a formation of spacecraft can be understood by studying the properties of the state transition matrix $\Phi$. This is the approach we will take throughout this paper.

\section{Stabilizing the Relative Motion}

The dynamics of long- and short-term relative motion are discussed more fully in Ref. 2, where we see that the relative motion of the spacecraft over a short time span centered at $t_{i}$ can be understood by analyzing the eigenvalues and eigenvectors of the matrix $A\left(t_{i}\right)$, as detailed in Ref. 5. Therefore, in deriving our control law we will use these short-term dynamics to guide our thinking.

Our goal is to stabilize relative motions in the sense of Lyapunov (not asymptotic stability), defined as follows ${ }^{10}$ : An equilibrium point $x_{e}$ of the system $\dot{x}=A(t) x$ is stable in the sense of Lyapunov if for any $\epsilon>0$, there exists a value $\delta\left(t_{0}, \epsilon\right)>0$ such that if $\| x\left(t_{0}\right)-$ $x_{e} \|<\delta$, then $\left\|x(t)-x_{e}\right\|<\epsilon$, regardless of $t$, where $t>t_{0}$.

In astrodynamics, stabilized trajectories will consist of oscillatory motions about the nominal trajectory. Consider Eq. (8) over a short time span centered at $t=t_{i}$, and reduce it to a second-order differential equation:

$$
\delta \ddot{\boldsymbol{r}}-2 \omega_{f} J \delta \dot{\boldsymbol{r}}-V_{\mathrm{RR}}\left(t_{i}\right) \delta \boldsymbol{r}=0
$$

To simplify the notation, we drop the time notation $t_{i}$ in the following discussion. A feedback controller $\mathcal{T}_{c}$ at time $t_{i}$ can be specified as

$$
\mathcal{T}_{c}=-V_{\mathrm{cv}} \delta \dot{\boldsymbol{r}}-V_{\mathrm{cr}} \delta \boldsymbol{r}
$$

so that the stabilized closed-loop equation at each time point is

$$
\delta \ddot{\boldsymbol{r}}-2 \omega_{f} J \delta \dot{\boldsymbol{r}}-V_{\mathrm{RR}} \delta \boldsymbol{r}+\mathcal{T}_{c}=0
$$

This is a nontraditional controller and is chosen to ensure a specific class of dynamical motions about the trajectory, not to drive a trajectory back to a target position. Thus, this controller should not be classified according to the usual rules of proportional, proportional derivative, etc.

Then the equations of motion in the feedback system are

$$
\delta \ddot{\boldsymbol{r}}-\left(2 \omega_{f} J+V_{\mathrm{cv}}\right) \delta \dot{\boldsymbol{r}}-\left(V_{\mathrm{RR}}+V_{\mathrm{cr}}\right) \delta \boldsymbol{r}=0
$$

Let

$$
S=2 \omega_{f} J+V_{\mathrm{cv}}, \quad \tilde{V}=V_{\mathrm{RR}}+V_{\mathrm{cr}}
$$

We can simplify Eq. (16) into the following form:

$$
\delta \ddot{\boldsymbol{r}}-S \delta \dot{\boldsymbol{r}}-\tilde{V} \delta \boldsymbol{r}=0
$$

If $V_{\mathrm{cv}}$ and $V_{\mathrm{cr}}$ are chosen skew symmetric and symmetric, respectively, the structure of this system is maintained, and $S$ and $\tilde{V}$ in Eq. (17) are skew symmetric and symmetric, respectively. Moreover, if we can find a condition under which relative motions are elliptic, the controller can then be obtained by simply subtracting the original Coriolis and force potential matrices from the desired ones. As this increases our choice for future orbit design, we first concentrate on finding the condition for stability rather than proposing a specific control law.

Assume $\tilde{V}$ in Eq. (17) is negative definite and consider a Lyapunov function $\mathcal{V}(\delta \boldsymbol{r}, \delta \dot{\boldsymbol{r}})$,

$$
\mathcal{V}(\delta \boldsymbol{r}, \delta \dot{\boldsymbol{r}})=-\frac{1}{2} \delta \boldsymbol{r}^{T} \tilde{V} \delta \boldsymbol{r}+\frac{1}{2} \delta \dot{\boldsymbol{r}}^{T} \delta \dot{\boldsymbol{r}}
$$

Then $\mathcal{V}(0)=0$ and $\mathcal{V}(\delta \boldsymbol{x})>0$ if $\delta \boldsymbol{x} \neq 0$, where $\delta \boldsymbol{x}=(\delta \boldsymbol{r}, \delta \dot{\boldsymbol{r}})$ (see the Appendix). Additionally,

$$
\begin{aligned}
\dot{\mathcal{V}}(\delta \boldsymbol{r}, \delta \dot{\boldsymbol{r}}) & =-\delta \dot{\boldsymbol{r}}^{T} \tilde{V} \delta \boldsymbol{r}+\delta \dot{\boldsymbol{r}}^{T} \delta \ddot{\boldsymbol{r}} \\
& =-\delta \dot{\boldsymbol{r}}^{T} \tilde{V} \delta \boldsymbol{r}+\delta \dot{\boldsymbol{r}}^{T} S \delta \dot{\boldsymbol{r}}+\delta \dot{\boldsymbol{r}}^{T} \tilde{V} \delta \boldsymbol{r} \\
& =\delta \dot{\boldsymbol{r}}^{T} S \delta \dot{\boldsymbol{r}} \\
& =0
\end{aligned}
$$

Because the requirement for Lyapunov stability is that the time derivative of a Lyapunov function be less than or equal to zero, our linear system is stable in the sense of Lyapunov. Also, because of its structure, this result implies that our local time-invariant system has all of its poles on the imaginary axis. This linear system can be represented as

$$
\begin{gathered}
\delta \dot{\boldsymbol{x}}=\tilde{A}\left(t_{i}\right) \delta \boldsymbol{x} \\
\tilde{A}\left(t_{i}\right)=\left[\begin{array}{ll}
0 & I \\
\tilde{V} & S
\end{array}\right]
\end{gathered}
$$

Given $V_{\mathrm{cv}}$ zero or skew symmetric and $V_{\mathrm{RR}}+V_{\mathrm{cr}}$ negative definite, the time-invariant dynamical system defined by Eq. (16) is stable. We note that this function does not guarantee stability of the time-varying system or of the nonlinear system. These issues were considered in more detail in Ref. 2.

An example of such a feedback control law was proposed in Ref. 2. To implement that control at a given time $t_{i}$, we evaluate the local eigenstructure of the matrix $A\left(t_{i}\right)$, find the characteristic exponents of the hyperbolic motion $\pm \sigma\left(t_{i}\right)$, and find the eigenvectors that define the stable and unstable manifolds of this motion $\boldsymbol{u}_{ \pm}\left(t_{i}\right)$, where the + denotes the unstable manifold and - denotes the stable one. Then the applied control acceleration is

$$
\mathcal{T}_{c}=-\sigma\left(t_{i}\right)^{2} G\left[\boldsymbol{u}\left(t_{i}\right)_{+} \boldsymbol{u}\left(t_{i}\right)_{+}^{T}+\boldsymbol{u}\left(t_{i}\right)_{-} \boldsymbol{u}\left(t_{i}\right)_{-}^{T}\right] \delta \boldsymbol{r}
$$

where $G$ is the gain parameter and $\delta \boldsymbol{r}$ is the measured relative position vector, that is, the offset between the periodic orbit and the spacecraft at time $t_{i}$. This control law maintains the structure of the problem and provides local and global stability if the gain constant $G$ is chosen sufficiently large.

Figures 1 and 2 provide two numerical examples. The controlled trajectory remains stable after two halo orbit periods (about one year), whereas the uncontrolled trajectory diverges after 66 days. In these two examples the initial position offsets are $1 \times 10^{5} \mathrm{~km}$, which is much larger than the mission requirements, usually $1 \sim 100 \mathrm{~km}$. In this example the linear control law just mentioned is used to control a spacecraft subject to nonlinear dynamics. By using an extremely large offset, we can verify that the controller performs well outside of the linear regime. Such simulations give us an indication of the robustness of this approach. After the control law in Eq. (22) is applied, the force potential becomes negative definite, as shown in Fig. 3.

\section{Overview on Trajectory Design}

\section{Objectives}

Our goal is to derive a feedback control law such that a spacecraft formation moving about an unstable trajectory can stabilize its motion and orbit in a circular or near-circular orbit relative to the trajectory with a fixed orientation in either the rotating or inertial frame. In Eq. (14), if $V_{\mathrm{cv}}$ and $V_{\mathrm{cr}}$ can be designed to generate such a specific class of motion relative to a nominal trajectory, they can be easily uploaded and distributed across the spacecraft, which then only require relative knowledge of their position or velocity to implement. We do not address system-wide control issues such as avoiding impact or close approaches. Our current approach is to specify the eigenstructure of the resultant controlled state transition matrix and then derive the general control law that will yield this motion. In this paper we want to design three general cases: A, fixed 




Fig. 1 Control law $T=-\sigma^{2} G\left[u_{+} u_{+}^{T}+u_{-} u_{-}^{T}\right] \delta r$ with $G=10$ is applied [Eq. (22)] Initial deviation is $1 \times 10^{5} \mathrm{~km}$ in the $x$ direction. This plot shows the stability in a nonlinear solution.



Fig. 2 Controller $\mathcal{T}_{c}=-\left(\tilde{V}_{\mathrm{rr}}-V_{\mathrm{rr}}\right) \delta r$ is applied to result in a desired force potential $\tilde{V}_{\mathrm{rr}}=-2.7 \times 10^{-12} \mathrm{I}_{3} \times 3$. Initial deviation is $1 \times 10^{5} \mathrm{~km}$ in the $x$ direction. This plot shows the stability in a nonlinear solution.

relative orbit plane in the original rotating frame; $\mathrm{B}$, fixed relative orbit plane in the inertial frame; and $\mathrm{C}$, fixed relative orbit plane in an arbitrary frame.

\section{Local Dynamics Formulation}

Having found a sufficient condition under which the closed-loop linearized system is stable, we now concern ourselves with the dynamics of the controlled system. In preceding sections, we approached the problem by considering stability at each point in time and treating the instantaneous dynamics over every short time span as time invariant instead of considering the original linearized timevarying system over an entire period. We can understand our system by investigating the eigenstructure of each $\tilde{A}\left(t_{i}\right)$ (stabilized) matrix.
For the case of a completely stable map, there are three pairs of imaginary eigenvalues, known as the modes of the system. The trajectory described by each mode forms an elliptical orbit relative to the nominal trajectory with the origin of the frame at its center. The actual trajectory is the linear combination of these three modes. Because $\tilde{A}\left(t_{i}\right)$ is not necessarily constant over the entire orbit period, we cannot define one set of constants describing the relative motion over the entire orbit period but can instead define a secular set of "linear orbital elements" 5 to describe the geometry of the trajectory.

By understanding the behavior of each mode over time, we can understand how the relative motion of the formation will be modified over time. Moreover, the mathematical formulas link the eigenvectors of the dynamic matrix with linear orbital elements, which converts this into a problem of eigenstructure properties. 


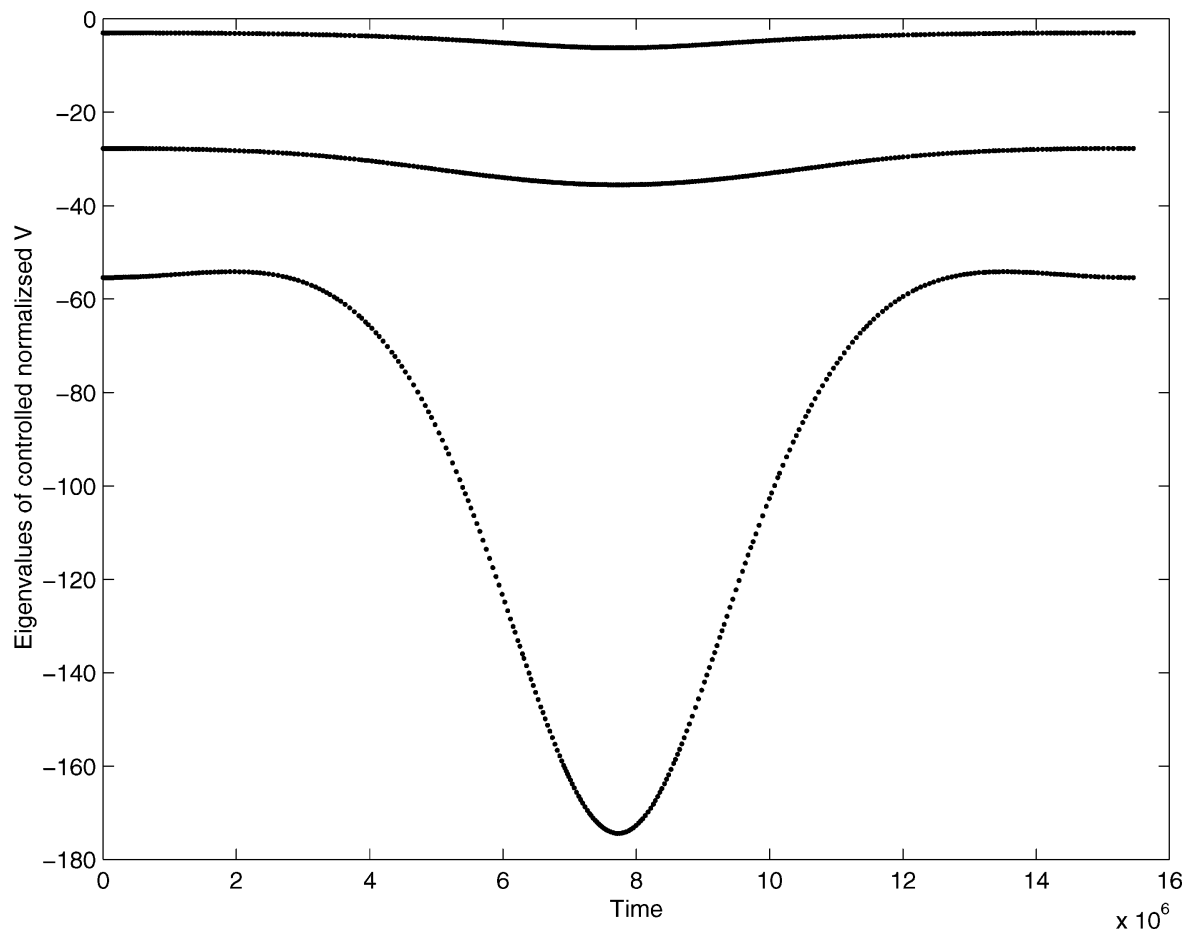

Fig. 3 Eigenvalues of control law in Eq. (22). We see that the $V$ is negative definite.

\section{Eigenstructure Assignment}

It is well known that, for a controllable system, we can arbitrarily place its poles. ${ }^{11}$ However, the desired closed-loop eigenvalues and eigenvectors might not be attainable at the same time because this is an overconstrained problem. This implies that closed-loop right eigenvectors cannot be assigned arbitrarily, and only those that belong to the span of the corresponding allowable subspace can be assigned precisely. ${ }^{12}$ Most researchers have dealt with this problem by using an optimization technique, such as Refs. 12 and 13, with either output feedback or state feedback. These algorithms try to "rotate" the subspace of eigenstructures such that the weighting is minimum. With certain constraints the system must also satisfy, stability can then be guaranteed.

This is a useful tool for control problems, which require poles on the negative real plane. In our problem, however, these approaches are not applicable. Our approach to formation flight requires that all instantaneous closed-loop poles should lie on the imaginary axis. Optimization of the eigenvector placement would in general move those poles off the imaginary axis, which would destabilize the formation dynamics. Therefore, we have investigated other approaches to solve our problem.

\section{Trajectory Design in the Rotating Frame}

\section{Position-Only Feedback: Algebraic Approach}

General Equations

To understand the relationship between eigenvalues and eigenvectors, recall Eq. (17) for a three-degree-of-freedom system with position-only feedback

$$
\delta \ddot{\boldsymbol{r}}-2 \omega_{f} J \delta \dot{\boldsymbol{r}}-\tilde{V}(t) \delta \boldsymbol{r}=0
$$

Therefore, the characteristic equation for Eq. (23) is

$$
\operatorname{det}\left[\lambda^{2} I-2 \omega_{f} J \lambda-\tilde{V}(t)\right]=0
$$

Given our stability constraint, the eigenvalues are $\lambda= \pm j \omega_{i}$, where $\omega_{i}$ is the closed-loop oscillation frequency for $i$ th mode and $i=1,2,3$. We can express those frequencies in terms of frame rotation rate $\omega_{f}$, that is, $\omega_{i}=k_{i} \omega_{f}$, which must satisfy Eq. (24). Define $\bar{V}(t)=\tilde{V}(t) / \omega_{f}^{2}$, and then the normalized characteristic equation would be

$$
\operatorname{det}\left[-k_{i}^{2} I-2 k_{i} j J-\bar{V}(t)\right]=0
$$

If $\boldsymbol{\alpha}_{i} \pm j \boldsymbol{\beta}_{i}$ are the associated eigenvectors for each mode in the normalized system at each time instant, the corresponding eigenvector problem can be stated as

$$
\left[\begin{array}{ccc}
-k_{i}^{2}-\bar{V}_{x x} & \mp 2 k_{i} j-\bar{V}_{x y} & -\bar{V}_{x z} \\
\pm 2 k_{i} j-\bar{V}_{x y} & -k_{i}^{2}-\bar{V}_{y y} & -\bar{V}_{y z} \\
-\bar{V}_{x z} & -\bar{V}_{y z} & -k_{i}^{2}-\bar{V}_{z z}
\end{array}\right]\left[\begin{array}{c}
a_{1 i} \pm j b_{1 i} \\
a_{2 i} \pm j b_{2 i} \\
a_{3 i} \pm j b_{3 i}
\end{array}\right]=0
$$

where $\boldsymbol{\alpha}_{i}=\left(a_{1 i}, a_{2 i}, a_{3 i}\right), \boldsymbol{\beta}_{i}=\left(b_{1 i}, b_{2 i}, b_{3 i}\right)$. Considering only one mode, we can rewrite Eq. (26) as a function of $\bar{V}_{i j}$.

$\left[\begin{array}{cccccc}a_{1} & a_{2} & a_{3} & 0 & 0 & 0 \\ b_{1} & b_{2} & b_{3} & 0 & 0 & 0 \\ 0 & a_{1} & 0 & a_{2} & a_{3} & 0 \\ 0 & b_{1} & 0 & b_{2} & b_{3} & 0 \\ 0 & 0 & a_{1} & 0 & a_{2} & a_{3} \\ 0 & 0 & b_{1} & 0 & b_{2} & b_{3}\end{array}\right]\left[\begin{array}{c}\bar{V}_{x x} \\ \bar{V}_{x y} \\ \bar{V}_{x z} \\ \bar{V}_{y y} \\ \bar{V}_{y z} \\ \bar{V}_{z z}\end{array}\right]=-k^{2}\left[\begin{array}{c}a_{1} \\ b_{1} \\ a_{2} \\ b_{2} \\ a_{3} \\ b_{3}\end{array}\right]-2 k\left[\begin{array}{c}-b_{2} \\ a_{2} \\ b_{1} \\ -a_{1} \\ 0 \\ 0\end{array}\right]$

which can also be expressed in the form of $\boldsymbol{P}_{i} \overline{\boldsymbol{V}}=\boldsymbol{Q}_{i}(\overline{\boldsymbol{V}}), i=1,2,3$. Because all three modes have to satisfy the same force potential, Eq. (27) is valid for all three modes. Let

$$
\begin{gathered}
\boldsymbol{P}=\left[\begin{array}{l}
\boldsymbol{P}_{1} \\
\boldsymbol{P}_{2} \\
\boldsymbol{P}_{3}
\end{array}\right] \\
\boldsymbol{Q}=\left[\begin{array}{l}
\boldsymbol{Q}_{1} \\
\boldsymbol{Q}_{2} \\
\boldsymbol{Q}_{3}
\end{array}\right]
\end{gathered}
$$

Then we can combine these three modes and write them as $\boldsymbol{P} \overline{\boldsymbol{V}}=\boldsymbol{Q}(\overline{\boldsymbol{V}})$, where $\boldsymbol{P} \in \mathbb{R}^{18 \times 6}$ and $\boldsymbol{Q} \in \mathbb{R}^{18}$. This is an overconstrained simultaneous nonlinear equation with six unknowns and 18 
equations. The solution exists if and only if $\boldsymbol{Q}(\overline{\boldsymbol{V}}) \in \mathcal{R} \mathcal{A}\{\boldsymbol{P}\}$. Both $\boldsymbol{P}$ and $\boldsymbol{Q}$ are functions of $k, \boldsymbol{\alpha}$, and $\boldsymbol{\beta}$, and $k$ in turn is also a function of $\overline{\boldsymbol{V}}$. Generally speaking, this problem is difficult to solve.

In a practical approach, we can pick a set of candidate eigenvectors and eigenvalues and insert them into Eqs. (25) and (27) to verify whether they satisfy both equations. To simplify the situation, we can only specify one mode and let the other two modes be free. Upon finding some candidate force potentials, we can then enforce the negative definite condition, if possible, so that stability is guaranteed.

\section{Forcing Circular Motion}

The most specific case to consider is the design of a circular relative trajectory. Following our preceding design method, we only consider one mode and pick the negative force potential later. From Ref. 5, we can conclude that $\boldsymbol{\alpha} \perp \boldsymbol{\beta} \perp \hat{\boldsymbol{h}}$ and $|\boldsymbol{\alpha}|=|\boldsymbol{\beta}|=1 / \sqrt{ } 2$ for a circular trajectory mode, where $\hat{\boldsymbol{h}}$ is the relative orbit normal vector. Assume $\boldsymbol{\alpha}=\left(\hat{a}_{1}, \hat{a}_{2}, \hat{a}_{3}\right) / \sqrt{ } 2, \boldsymbol{\beta}=\left(\hat{b}_{1}, \hat{b}_{2}, \hat{b}_{3}\right) / \sqrt{ } 2$, and $\hat{\boldsymbol{h}}=$ $\left(h_{1}, h_{2}, h_{3}\right)$, where $\hat{a}_{i}$ and $\hat{b}_{i}$ denote the unit vector components of $\boldsymbol{\alpha}$ and $\boldsymbol{\beta}$. Then $\hat{\boldsymbol{h}}=2(\boldsymbol{\beta} \times \boldsymbol{\alpha})$. Accordingly,

$$
\begin{aligned}
\hat{\alpha} & =\hat{\boldsymbol{h}} \times \hat{\beta} \\
& =\left[\begin{array}{l}
h_{2} \hat{b}_{3}-h_{3} \hat{b}_{2} \\
h_{3} \hat{b}_{1}-h_{1} \hat{b}_{3} \\
h_{1} \hat{b}_{2}-h_{2} \hat{b}_{1}
\end{array}\right] \\
\hat{\beta} & =\hat{a} \times \hat{\boldsymbol{h}} \\
& =\left[\begin{array}{l}
-h_{2} \hat{a}_{3}+h_{3} \hat{a}_{2} \\
-h_{3} \hat{a}_{1}+h_{1} \hat{a}_{3} \\
-h_{1} \hat{a}_{2}+h_{2} \hat{a}_{1}
\end{array}\right]
\end{aligned}
$$

Substituting these into Eq. (27) and performing some algebraic manipulation leads to two relations:
The expansion of Eq. (33) yields

$$
2 h_{1} h_{2} k\left(k^{2}+\bar{V}_{z z}\right)\left(-\bar{V}_{x x}+\bar{V}_{y y}\right)=0
$$

One of the possible solutions in Eq. (34) is $k^{2}+\bar{V}_{z z}=0$, which is consistent with Eq. (32). However, because we know the rank of Eqs. (32) and (33) are not greater than one, this is not a qualified solution. The other solution is $\bar{V}_{x x}=\bar{V}_{y y}$, and, from Eq. (32), $\bar{V}_{x x}=\bar{V}_{y y}=-\left(k^{2}+2 k h_{3}\right)$. Therefore, $\hat{\beta}=(1,0,0), \hat{\alpha}=(0,1,0)$, and $\hat{\boldsymbol{h}}=(0,0,1)$. On the other hand, $-k^{2}=\bar{V}_{x x}+2 k=\bar{V}_{y y}+2 k$. We find that the characteristic equation, Eq. (25), is also satisfied. Moreover, Eqs. (32) and (33) both degenerate to rank $\leq 1$ with these results, which shows the consistency in our derivation. This result implies that, with the presence of a Coriolis force, circular relative motion can only exist in the $x-y$ plane; a numerical simulation is shown in Fig. 4. This restricts the application of this control law to interferometric imaging, where we wish to orient the formation plane in an arbitrary direction.

In Ref. 7 we also develop a so-called "soft approach" to approximate trajectory design. This algorithm provides a simpler method to perform a trajectory design with position-only feedback. Relatively large errors can exist between the true and desired trajectories with this approach, which also restricts the application. Thus we do not consider it further in the current paper.

\section{Position-and-Velocity Feedback}

The difficulties experienced in our trajectory design in the preceding section is caused by the presence of the Coriolis force. To create a non-Coriolis environment in the rotational system, we must apply a velocity feedback term to null out the Coriolis force:

$$
\mathcal{T}_{c}=2 \omega_{f} J \delta \dot{\boldsymbol{r}}-V_{\mathrm{cr}}(t) \delta \boldsymbol{r}
$$

Then the stabilized equations of motion are in the form

$$
\delta \ddot{\boldsymbol{r}}-\tilde{V}(t) \delta \boldsymbol{r}=0
$$

$$
\begin{gathered}
{\left[\begin{array}{ccc}
k^{2}+2 k h_{3}+\bar{V}_{x x} & \bar{V}_{x y} & -2 k h_{1}+\bar{V}_{x z} \\
\bar{V}_{x y} & k^{2}+2 k h_{3}+\bar{V}_{y y} & -2 k h_{2}+\bar{V}_{y z} \\
\bar{V}_{x z} & \bar{V}_{y z} & k^{2}+\bar{V}_{z z}
\end{array}\right] \boldsymbol{\alpha}=0} \\
{\left[\begin{array}{ccc}
-\bar{V}_{x y} h_{3}+\bar{V}_{x z} h_{2} & \left(k^{2}+\bar{V}_{x x}\right) h_{3}-\bar{V}_{x z} h_{1}+2 k & -\left(k^{2}+\bar{V}_{x x}\right) h_{2}+\bar{V}_{x y} h_{1} \\
-\left(k^{2}+\bar{V}_{y y}\right) h_{3}+\bar{V}_{y z} h_{2}-2 k & \bar{V}_{x y} h_{3}-\bar{V}_{y z} h_{1} & -\bar{V}_{x y} h_{2}+\left(k^{2}+\bar{V}_{y y}\right) h_{1} \\
-\bar{V}_{y z} h_{3}+\left(k^{2}+\bar{V}_{z z}\right) h_{2} & \bar{V}_{x z} h_{3}-\left(k^{2}+\bar{V}_{z z}\right) h_{1} & -\bar{V}_{x z} h_{2}+\bar{V}_{y z} h_{1}
\end{array}\right] \boldsymbol{\alpha}=0}
\end{gathered}
$$

Note that $\boldsymbol{\beta}$ also has to satisfy Eqs. (30) and (31). Because $\boldsymbol{\alpha}$ is perpendicular to $\boldsymbol{\beta}$, the rank of Eqs. (30) and (31) cannot be greater than one, and $\boldsymbol{\alpha}$ and $\boldsymbol{\beta}$ must be located in the null space of both matrices.

Because the matrices in Eqs. (30) and (31) are not full rank, by taking their determinants we can obtain relations between the factor $k$ and the entries of $\bar{V}$. Consider a specific case in which the offdiagonal terms in $\bar{V}$ are all zero. The determinants are

$$
\begin{gathered}
\left|\begin{array}{ccc}
k^{2}+2 k h_{3}+\bar{V}_{x x} & 0 & -2 k h_{1} \\
0 & k^{2}+2 k h_{3}+\bar{V}_{y y} & -2 k h_{2} \\
0 & 0 & k^{2}+\bar{V}_{z z}
\end{array}\right|=0 \quad \text { (32) } \\
\left|\begin{array}{ccc}
\left.\mid k^{2}+\bar{V}_{x x}\right) h_{3}+2 k & -\left(k^{2}+\bar{V}_{x x}\right) h_{2} \\
-\left(k^{2}+\bar{V}_{y y}\right) h_{3}-2 k & 0 & \left(k^{2}+\bar{V}_{y y}\right) h_{1} \\
\left(k^{2}+\bar{V}_{z z}\right) h_{2} & -\left(k^{2}+\bar{V}_{z z}\right) h_{1} & 0
\end{array}\right|=0
\end{gathered}
$$

If we assume $\tilde{V}(t)$ is constant over a short time interval, we can easily derive the "instantaneous" motion. The characteristic equation is

$$
\begin{aligned}
\operatorname{det}\left(\omega^{2} I-\tilde{V}\right) & =\operatorname{det}(M) \operatorname{det}\left(\omega^{2} I-\Lambda_{V}\right) \operatorname{det}\left(M^{-1}\right) \\
& =\operatorname{det}\left(\omega^{2} I-\Lambda_{V}\right) \\
& =\left(\omega^{2}-\lambda_{v 1}\right)\left(\omega^{2}-\lambda_{v 2}\right)\left(\omega^{2}-\lambda_{v 3}\right) \\
& =0
\end{aligned}
$$

where $M$ is the eigenvector matrix and $\Lambda_{V}$ is the diagonalized force potential. From linear theory, the force potential can be decomposed as $\tilde{V}=M \Lambda_{V} M^{-1}$, and the eigenvectors satisfy the equation

$$
\left[\omega_{i}^{2} I-\tilde{V}\right] \boldsymbol{u}_{i}=0
$$

Here we should note that the controlled $\tilde{V}$ is negative definite for stabilization purposes, implying that $\lambda_{\mathrm{vi}}<0$. Given this local 


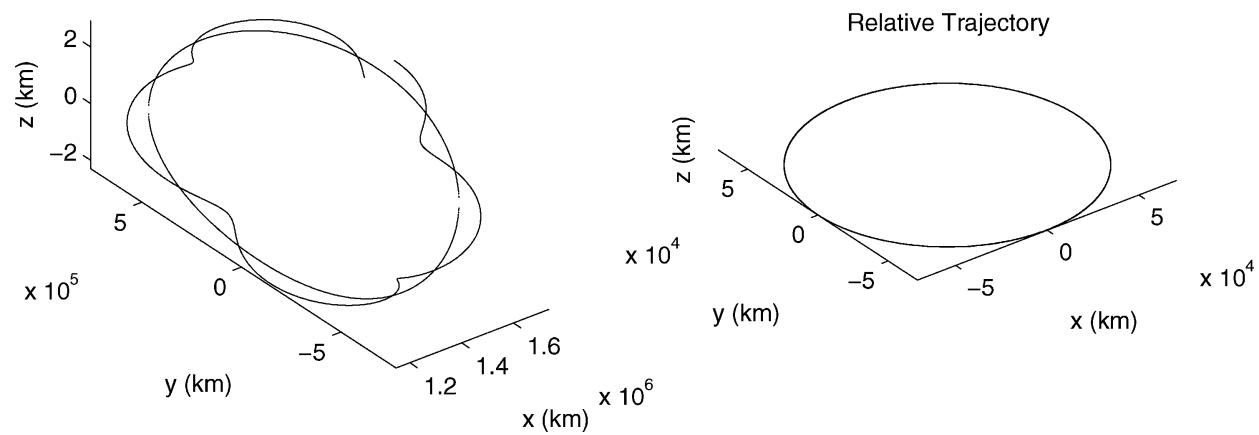

Fig. $4 \quad V_{x x}=V_{y y}$ and off-diagonal term being zeros are selected in this case. We can see that the relative circular trajectory only can exist on the $x y$ plane; a) integrated in the original nonlinear equations about a halo orbit and b) same orbit relative to the halo orbit in panel a.

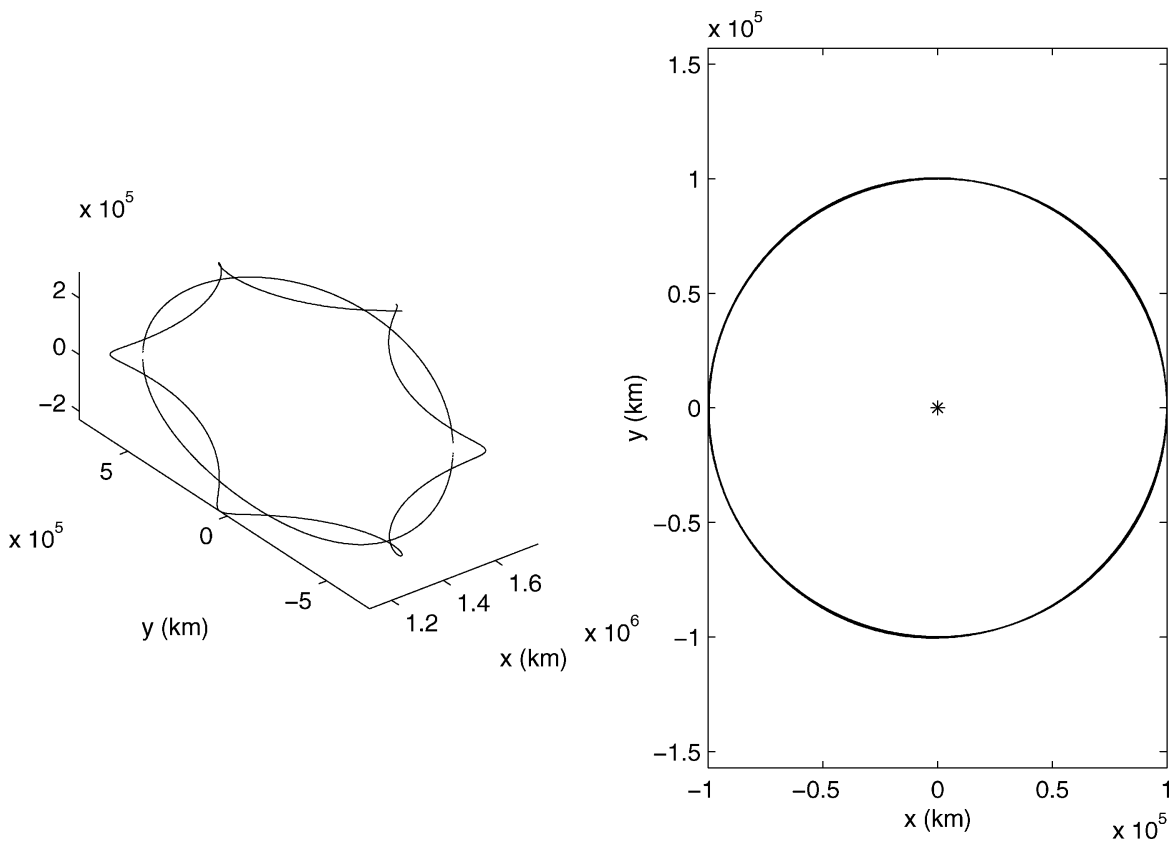

Fig. 5 Relative circular trajectory is created in a non-Coriolis system: a) integrated in the original nonlinear equations about a halo orbit and b) same orbit relative to the halo orbit in panel a.

description, we can solve for the motion (assuming local time invariance)

$$
\delta \boldsymbol{r}(t)=\sum_{i=1}^{3} \rho_{i} \boldsymbol{u}_{i} \cos \left(\sqrt{\left|\lambda_{\mathrm{vi}}\right|} t+\phi_{i}\right)
$$

where $\rho_{i}$ and $\phi_{i}$ are determined by initial conditions. This equation tells us that, for each mode, the relative trajectory performs linear harmonic oscillations along the direction of the eigenvector with their associated eigenvalue as their frequency. The real trajectory is just the linear combination of all three modes. Moreover, all of the eigenvectors are orthonormal to each other because $\tilde{V}$ is symmetric, and so the eigenvector matrix $M$ can be viewed as a rotation matrix. To generate a desired trajectory, we can initially assemble a diagonal $\tilde{V}$ matrix with proper frequency ratios and then choose initial conditions to generate a desired relative trajectory. Following this, we apply frame rotation by pre- and postmultiplication by a rotation matrix to obtain a specific orientation of the orbit plane.

Figures 5 and 6 show an example of mode combinations, where a circular and a figure-eight shaped trajectory are created, indicating that we now have considerable freedom to perform trajectory design in this way. In general, if the frequencies are chosen to be commensurate with each other, the resulting trajectories will be periodic. For our motivational application of interferometric imaging, we are most interested in circular orbits; hence, we will generally consider equal frequencies with initial conditions chosen to excite the spacecraft into a circular orbit.

\section{General Trajectory Design}

The preceding discussion shows the convenience of designing a trajectory with position-and-velocity feedback. The philosophy behind this control law is to create artificial inertial frames, where all of the motions are just combinations of simple harmonic oscillations. Having accomplished our first goal of trajectory design - a fixed relative orbit plane in the sun-Earth rotating frame-in this section we explore how to achieve our other two goals: a fixed relative orbit plane in the inertial frame and plane reorientation.

\section{Transformation of Control Laws}

We first discuss the transformation of control laws between frames in uniform rotation relative to each other. In previous derivations we have developed control laws with respect to the sun-Earth rotational system; thus, to be consistent when designing our formation controls we transform them back to the sun-Earth rotational system.

To generalize the derivation, we define three different systems:

1) The first is the inertial frame with all notations denoted by the subscript $i$. 

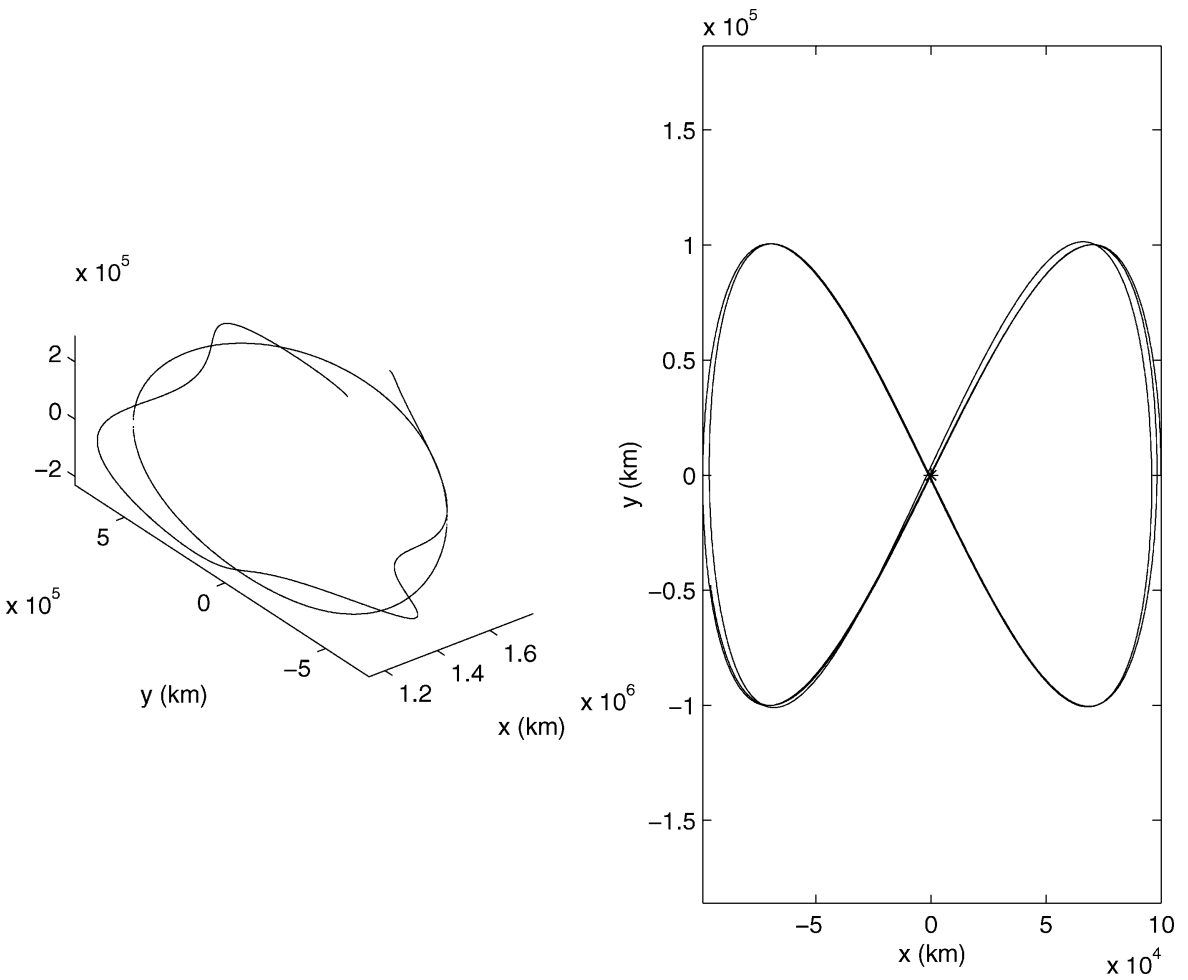

Fig. 6 Relative figure-eight trajectory in a non-Coriolis system: a) integrated in the original nonlinear equations about a halo orbit and b) same orbit relative to the halo orbit in panel a.

2) The second is the $A$-rotational frame with all notations denoted by the subscript $A$. Assume that the $A$ frame rotates with rotational vector $\boldsymbol{\omega}_{A}=\omega_{A} \hat{z}$ with respect to the inertial frame.

3) The last system is the $B$-rotational frame with all notations denoted by the subscript $B$. Assume that the $B$ frame rotates with rotational vector $\omega_{B}=\omega_{B} \hat{u}$, where $\hat{u}$ is an arbitrary unit vector, with respect to the inertial frame. We note that the sun-Earth system is an $A$-frame system with rotational vector $\boldsymbol{\omega}_{e}=\omega_{e} \hat{z}$.

Next we consider a general transformation of control laws between a rotational frame with rotational vector $\boldsymbol{\omega}=\left[\begin{array}{lll}\omega_{x} & \omega_{y} & \omega_{z}\end{array}\right]^{T}$ and an inertial frame. Assume the excursion of a spacecraft in the rotating frame is described as $\boldsymbol{r}_{r}(t)$ (where the $\delta$ sign is dropped for convenience), and its excursion in the inertial frame is $\boldsymbol{r}_{i}(t)$. The excursions in the inertial frame can be transformed by a rotation matrix to $\boldsymbol{r}_{r}(t)=R(t) \boldsymbol{r}_{i}(t)$ and $\dot{R}=-\tilde{\omega} R$, where $\tilde{\omega}$ is the outer product operator with $\boldsymbol{\omega}=\left(\omega_{x}, \omega_{y}, \omega_{z}\right)$, and

$$
\tilde{\omega}=\left[\begin{array}{ccc}
0 & -\omega_{z} & \omega_{y} \\
\omega_{z} & 0 & -\omega_{x} \\
-\omega_{y} & \omega_{x} & 0
\end{array}\right]
$$

Therefore, the following equations can be obtained:

$$
\begin{aligned}
\boldsymbol{r}_{r}(t) & =R(t) \boldsymbol{r}_{i}(t) \\
\dot{\boldsymbol{r}}_{r}(t) & =\dot{R}(t) \boldsymbol{r}_{i}(t)+R(t) \dot{\boldsymbol{r}}_{i}(t) \\
& =-\tilde{\omega} R(t) \boldsymbol{r}_{i}(t)+R(t) \dot{\boldsymbol{r}}_{i}(t) \\
\ddot{\boldsymbol{r}}_{r}(t) & =\tilde{\omega} \tilde{\omega} R(t) \boldsymbol{r}_{i}(t)-2 \tilde{\omega} R(t) \dot{\boldsymbol{r}}_{i}(t)+R(t) \ddot{\boldsymbol{r}}_{i}(t)
\end{aligned}
$$

where $\dot{\boldsymbol{r}}_{r}$ and $\ddot{\boldsymbol{r}}_{r}$ are the velocity and acceleration vectors observed in the rotational frame. In our notation time derivatives are taken with respect to the frame specified in the subscript; thus, $\dot{\boldsymbol{r}}_{r}$ is with respect to the rotating frame and $\dot{\boldsymbol{r}}_{i}$ is with respect to the inertial frame. Because $R(t) \boldsymbol{r}_{i}(t)=\boldsymbol{r}_{r}(t)$ and $R(t) \dot{\boldsymbol{r}}_{i}(t)=\dot{\boldsymbol{r}}_{r}(t)+\tilde{\omega} \boldsymbol{r}_{r}(t)$ [Eqs. (41) and (42)], Eq. (43) can be reformulated as

$$
\ddot{\boldsymbol{r}}_{r}(t)=-\tilde{\omega} \tilde{\omega} \boldsymbol{r}_{r}(t)-2 \tilde{\omega} \dot{\boldsymbol{r}}_{r}(t)+R(t) \ddot{\boldsymbol{r}}_{i}(t)
$$

Once we specify the desired equations of motion for our formation in the inertial frame, Eqs. (43) and (44) allow us to transform them back to the rotational frame. Given a rotation axis about which to rotate $\hat{u}$ and a rotation angle $\phi=\omega t$, we note that the rotation matrix $R(t)$ can be obtained from

$$
R(t)=\hat{u} \hat{u}^{T}+\cos \phi(t)\left(I-\hat{u} \hat{u}^{T}\right)-\sin \phi(t) \tilde{u}
$$

where $\tilde{u}$ is the outer product operator [Eq. (40)] of $\hat{u}$.

One immediate application of the preceding equations is to transform a control law, which generates a desired motion in the inertial frame into the $A$ frame. Assuming that the desired equations of motion in the inertial frame are given as $\ddot{\boldsymbol{r}}_{i}(t)=V_{i}(t) \boldsymbol{r}_{i}$, where $V_{i}$ is the force potential matrix in the inertial frame, we substitute this into Eq. (44) and apply $R(t) \boldsymbol{r}_{i}(t)=\boldsymbol{r}_{A}(t)$ to obtain

$$
\ddot{\boldsymbol{r}}_{A}(t)=-\tilde{\omega}_{A} \tilde{\omega}_{A} \boldsymbol{r}_{A}(t)-2 \tilde{\omega}_{A} \dot{\boldsymbol{r}}_{A}(t)+R(t) V_{i}(t) R^{T}(t) \boldsymbol{r}_{A}(t)
$$

Replacing the subscript $A$ with $e$ in Eq. (46), we obtain the required transformation for the sun-Earth system:

$$
\ddot{\boldsymbol{r}}_{e}(t)=-\tilde{\omega}_{e} \tilde{\omega}_{e} \boldsymbol{r}_{e}(t)-2 \tilde{\omega}_{e} \dot{\boldsymbol{r}}_{e}(t)+R(t) V_{i}(t) R^{T}(t) \boldsymbol{r}_{e}(t)
$$

Comparing Eq. (47) with Eq. (16), we see that the required controller $V_{\mathrm{cv}} \dot{\boldsymbol{r}}_{r}(t)+V_{\mathrm{cr}} \boldsymbol{r}_{r}(t)$ in Eq. (14) is

$$
\begin{gathered}
V_{\mathrm{cv}}(t)=-2 \tilde{\omega}_{e}-2 \omega_{e} J \\
V_{\mathrm{cr}}(t)=R(t) V_{i}(t) R^{T}(t)-\tilde{\omega}_{e} \tilde{\omega}_{e}-V_{\mathrm{RR}}(t)
\end{gathered}
$$

where

$$
\begin{aligned}
\tilde{\omega}_{e} & =\left[\begin{array}{ccc}
0 & -\omega_{e} & 0 \\
\omega_{e} & 0 & 0 \\
0 & 0 & 0
\end{array}\right] \\
& =-\omega_{e} J
\end{aligned}
$$

Therefore we obtain

$$
V_{\mathrm{cv}}(t)=0
$$




$$
\begin{aligned}
V_{\mathrm{cr}}(t) & =R(t) V_{i}(t) R^{T}(t)-\omega_{e}^{2} J J-V_{\mathrm{RR}}(t) \\
& =V_{I}(t)-\omega_{e}^{2} J J-V_{\mathrm{RR}}(t)
\end{aligned}
$$

where $V_{I}(t)=R(t) V_{i}(t) R^{T}(t)$. Equations (51) and (52) can be explained as follows. If we observe motion in the rotational frame, it will be affected by the Coriolis and centripetal forces. Conversely, if we wish to control an inertial motion in the rotational frame, we should keep the Coriolis force but subtract the centripetal force, which is why $V_{\mathrm{cv}}=0$ but $V_{\mathrm{cr}}$ has an additional term $\omega_{e}^{2} J J$.

Next, we consider a desired motion governed by $\ddot{\boldsymbol{r}}_{B}(t)=$ $V_{B}(t) \boldsymbol{r}_{B}(t)$ in the $B$ frame, where $V_{B}(t)$ is symmetric and represents the force potential. We have already shown how to force the relative orbit plane to be fixed in the rotational frame. Moreover, we know that a fixed trajectory in a rotational frame appears to be rotating in the inertial frame. Therefore, if we want to perform a trajectory plane reorientation with respect to an inertial frame we can fix the trajectory in a rotational frame until the trajectory plane reaches the desired inertial orientation.

To obtain the required control law, we substitute the preceding equations of motion into Eq. (43), with $R(t) \boldsymbol{r}_{i}(t)=\boldsymbol{r}_{B}(t)$ and $\dot{\boldsymbol{r}}_{B}(t)=R(t) \dot{\boldsymbol{r}}_{i}(t)-\tilde{\omega}_{B} R(t) \boldsymbol{r}_{i}(t)$, and we obtain

$$
\ddot{\boldsymbol{r}}_{i}(t)=2 R^{T}(t) \tilde{\omega}_{B} R \dot{\boldsymbol{r}}_{i}(t)+R^{T}(t)\left[V_{B}(t)-\tilde{\omega}_{B} \tilde{\omega}_{B}\right] R(t) \boldsymbol{r}_{i}(t)
$$

We can specify the required controller in the inertial frame as

$$
\begin{gathered}
V_{\mathrm{cv}}(t)=2 R^{T}(t) \tilde{\omega}_{B} R(t) \\
V_{\mathrm{cr}}(t)=R^{T}(t)\left[V_{B}(t)-\tilde{\omega}_{B} \tilde{\omega}_{B}\right] R(t)-V_{i}(t)
\end{gathered}
$$

\section{Trajectory Plane Reorientation}

To perform a trajectory plane reorientation, we use our ability to fix a plane in an arbitrary rotating frame, which results in a constant rotation of the plane in the inertial frame. When the desired orientation is reached, we switch the controller to resume the original dynamics.

This algorithm can be applied to a rotating frame as well. In the preceding position-and-velocity feedback section, we created a pseudo-inertial frame in the sun-Earth rotating frame by nulling out the Coriolis force. Therefore, to apply the preceding algorithm we can treat our sun-Earth system as an inertial frame by simply adding an additional controller $\mathcal{T}_{c 1}(t)=-2 \omega_{e} J \dot{\boldsymbol{r}}(t)$.

Consider the following scenario: A spacecraft formation is placed in a fixed circular trajectory relative to the periodic orbit in the sun-Earth rotating frame with its orientation defined by its relative angular momentum vector $\boldsymbol{h}_{b}$. It is desired to reorient the formation to reach a new plane defined by the angular momentum vector $\boldsymbol{h}_{a}$. To carry out this reorientation, the formation plane should be rotated about a rotation axis $\hat{u}$ defined by the intersection of the two planes, and obtained from the relation

$$
\hat{u}=\left(\boldsymbol{h}_{b} \times \boldsymbol{h}_{a}\right) /\left|\boldsymbol{h}_{b} \times \boldsymbol{h}_{a}\right|
$$

Therefore, the rotation vector is $\boldsymbol{\omega}=\omega \hat{u}$, where $\omega$ is arbitrary and is chosen according to how rapidly the formation should change its orbit plane orientation. After the desired plane is reached, the control law shifts back to its original form, and a fixed circular trajectory is resumed again.

To execute a plane reorientation with respect to the sun-Earth rotational system by applying Eq. (53), we first add an additional velocity feedback $\mathcal{T}_{c 1}(t)=-2 \omega_{e} J \dot{\boldsymbol{r}}(t)$ as mentioned earlier to generate a pseudo-inertial frame, and then apply Eq. (35) to obtain a fixed circular trajectory in the $B$ frame, which rotates about $\omega_{B}$ with respect to the sun-Earth pseudo-inertial frame. The modified controller is

$$
\begin{gathered}
V_{\mathrm{cv}}(t)=2 R^{T}(t) \tilde{\omega} R(t)-2 \omega_{e} J \\
V_{\mathrm{cr}}(t)=R^{T}(t)\left[V_{r}(t)-\tilde{\omega} \tilde{\omega}\right] R(t)-V_{\mathrm{RR}}(t)
\end{gathered}
$$

When the desired orientation is reached, Eq. (35) is applied again to fix the trajectory in its new orientation.

Figure 7 shows a numerical example of this operation. In this example, we apply a position-and-velocity feedback in Eq. (35) to create a circular trajectory. The original angular momentum $\boldsymbol{h}_{b}$ is chosen with inclination of $30^{\circ}$ and longitude of $90^{\circ}$ (facing the $y$
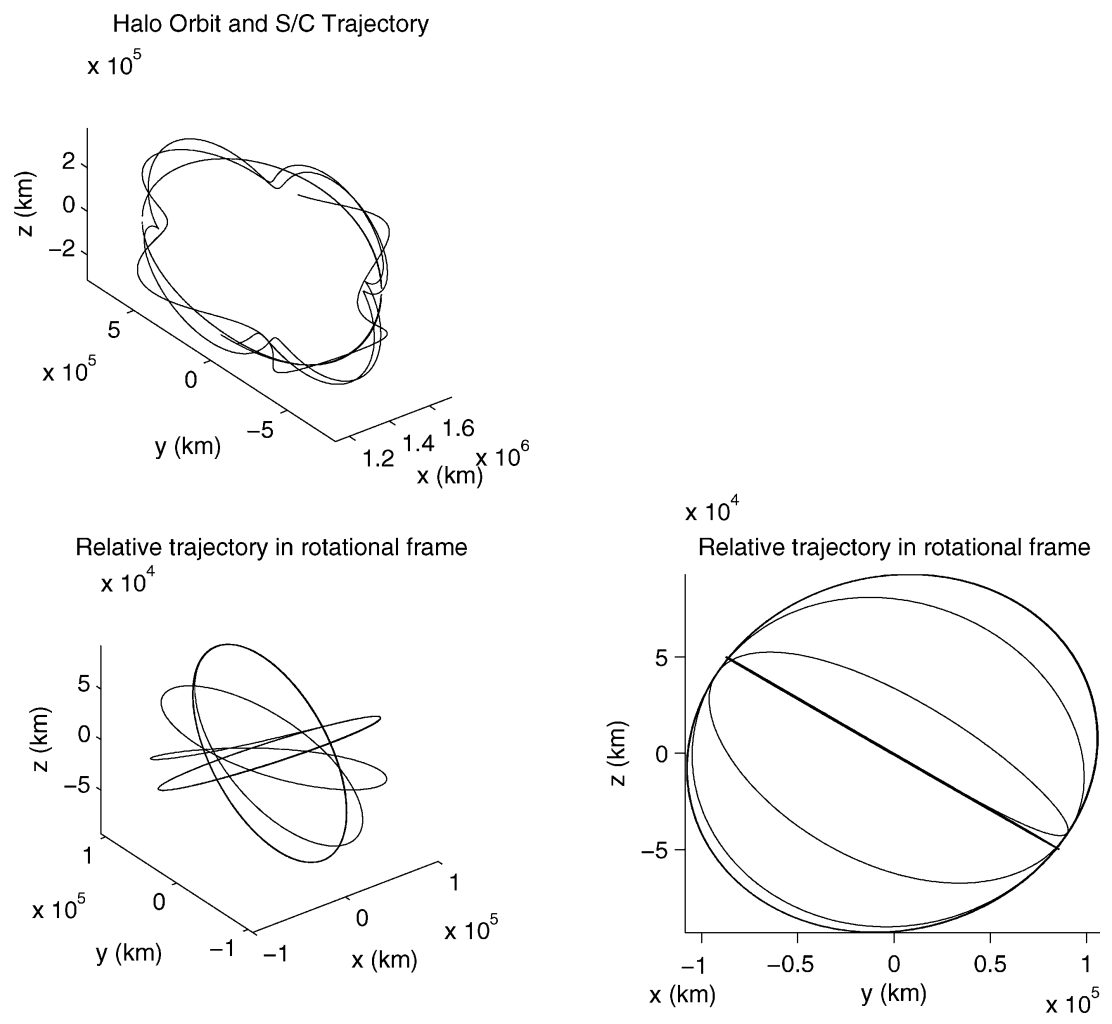

Fig. 7 Plane reorientation is demonstrated in this example. The final trajectory faces the $x$ axis as designed. However, because of the nonlinear effect, the final trajectory is not purely circular. 
axis). Given a new plane with $\boldsymbol{h}_{a}=\hat{x}$, we can apply Eqs. (56-58) to induce a plane reorientation. After the desired orientation is reached, Eq. (35) is applied again. In Fig. 7 we can clearly see that the final trajectory faces the $x$ axis. However, because of nonlinear effects, the final trajectory is not purely circular.

\section{Fixed Trajectory in the Inertial Frame}

Another important application of our preceding derivation is to create a circular trajectory relative to a halo orbit with fixed orientation in the inertial frame. Because our original equations of motion were derived in the sun-Earth system, a rotating frame, we would like to convert the necessary control law to this frame, which is already shown in Eqs. (51) and (52). This, however, makes the control law degenerate to a position-only feedback, and the equations of motion are similar to Eq. (23):

$$
\delta \ddot{\boldsymbol{r}}-2 \omega_{e} J \delta \dot{\boldsymbol{r}}-\left[V_{I}(t)-\omega_{e}^{2} J J\right] \delta \boldsymbol{r}=0
$$

but are still different from our preceding discussion. In Eq. (52) we cancel the centripetal force so that the trajectory will remain fixed in the inertial frame, whereas, in Eq. (23) the force potential is arbitrarily assigned. Recall that we originally desired a fixed circular trajectory in the rotating frame using position feedback only. However as shown in our earlier derivation, only one specific orbit plane orientation could achieve this goal. With the current result we see that a fixed trajectory in the inertial frame can be achieved with position-only feedback control. We note that this orbit will be uniformly rotating in the frame rotating with the Earth-sun line.

Figure 8 shows a numerical simulation of a fixed relative orbit in the inertial space. We see that the trajectory retains a constant orientation in the inertial frame. Combined with the preceding plane reorientation control, we can now easily switch a spacecraft formation from one fixed inertial orientation to another, all as they travel about the moving nominal spacecraft trajectory. The spacecraft formation relative orbit will, in general, maintain its same shape and size as it traverses to its new orbit plane.

\section{Cost of Implementation}

\section{Cost of the Various Approaches}

One important factor for the realizability of a control law is its cost. Here we define cost as the integral of control acceleration over time. Assuming that the greatest oscillation frequency in the system is $\omega_{\max }$, the total cost of the control laws we propose can be bounded as

$$
\begin{aligned}
|\Delta \boldsymbol{V}|= & \int_{0}^{t}|\boldsymbol{a}(t)| \mathrm{d} \tau \\
= & \int_{0}^{t} \mid V_{\mathrm{cv}} \delta \dot{\boldsymbol{r}}+V_{\mathrm{cr}} \delta \boldsymbol{r} \mathrm{d} \tau \\
\leq & \int_{0}^{t}\left(\left|V_{\mathrm{cv}} \delta \dot{\boldsymbol{r}}\right|+\left|V_{\mathrm{cr}} \delta \boldsymbol{r}\right|\right) \mathrm{d} \tau \\
\leq & \int_{0}^{t}\left(\omega_{\max }\left\|V_{\mathrm{cv}}\right\|_{2}+\left\|V_{\mathrm{cr}}\right\|_{2}\right)|\delta \boldsymbol{r}| \mathrm{d} \tau \\
\leq & \int_{0}^{t}\left(\omega_{\max }\left\|V_{\mathrm{cv}}\right\|_{2}+\left\|V_{\mathrm{cr}}\right\|_{2}\right)\left(\omega_{\max }\left\|\Phi_{\mathrm{rv}}\right\|_{2}+\left\|\Phi_{\mathrm{rr}}\right\|_{2}\right)\left|\delta \boldsymbol{r}_{0}\right| \mathrm{d} \tau \\
\leq & \left|\delta \boldsymbol{r}_{0}\right|\left[\int _ { 0 } ^ { t } ( \omega _ { \operatorname { m a x } } \| V _ { \mathrm { cv } } \| _ { 2 } + \| V _ { \mathrm { cr } } \| _ { 2 } ) \left(\omega_{\max }\left\|\Phi_{\mathrm{rv}}\right\|_{2}\right.\right. \\
& \left.\left.+\left\|\Phi_{\mathrm{rr}}\right\|_{2}\right) \mathrm{~d} \tau\right]
\end{aligned}
$$

where $\|\cdot\|_{2}$ is the 2-norm of a matrix, defined as $\sqrt{ }\left[\lambda_{\max }\left(A A^{T}\right)\right]$, where $\lambda_{\max }$ denotes the maximum eigenvalue of $A A^{T}$, and $\Phi$ is the state transition matrix with

$$
\Phi(t, 0)=\left[\begin{array}{l|l}
\Phi_{\mathrm{rr}} & \Phi_{\mathrm{rv}} \\
\hline \Phi_{\mathrm{vr}} & \Phi_{\mathrm{vv}}
\end{array}\right]
$$

We have discussed a variety of ways to design a formation: algebraic approach, soft approach, position-and-velocity feedback,



Relative trajectory in rotational frame

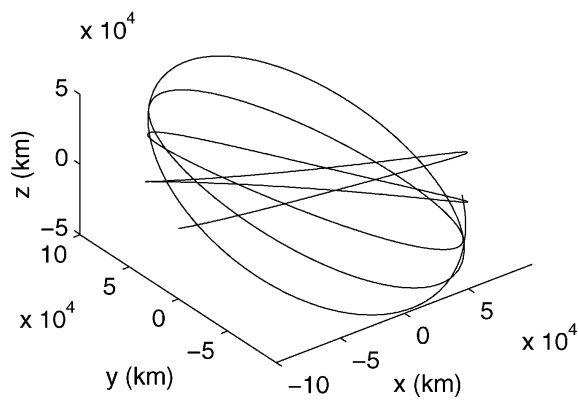

Relative trajectory in inertial frame

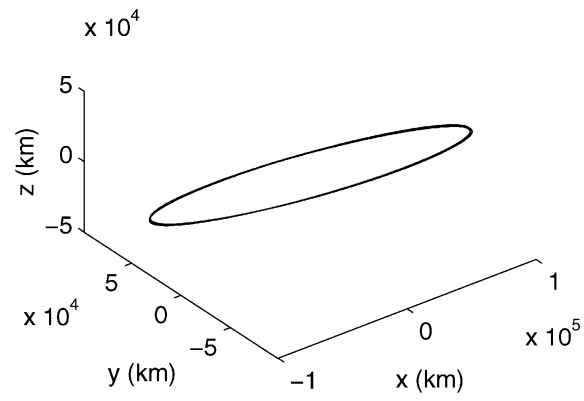

Fig. 8 Numerical simulation about fixed circular trajectory design in the inertial frame. 
plane reorientations, and fixed orbit planes in the inertial frame. With the preceding definition we can examine the costs of these approaches. Figures 9 and 10 provide numerical simulations of fuel usage in these approaches. All of them offer information on the upper bound of fuel usage and actual costs in a specific case.

Table 1 gives a general comparison of costs for the different operations shown in Figs. 9 and 10. The position-and-velocity feedback has more cost than the position-only controller because we need to spend fuel to null out the Coriolis force. The inertially fixed circular trajectory costs more because fuel is spent generating rotational motion in the original rotating frame. However, these levels of fuel con-

Table 1 Acceleration and fuel bounds for various control strategies for generating circular orbits with period $\sim 36$ days

\begin{tabular}{lcc}
\hline \hline Type of control law & $\leq \boldsymbol{a},{ }^{\mathrm{a}} \mathrm{km} / \mathrm{s}^{2}$ & $\Delta V / \mathrm{yr},{ }^{\mathrm{a}} \mathrm{km} / \mathrm{s} / \mathrm{yr}$ \\
\hline Position only & $4.30 \times 10^{-12}$ & $1.36 \times 10^{-4}$ \\
Soft approach & $4.98 \times 10^{-12}$ & $1.57 \times 10^{-4}$ \\
Position and velocity & $4.66 \times 10^{-12}$ & $1.47 \times 10^{-4}$ \\
Inertially fixed trajectory & $5.81 \times 10^{-12}$ & $1.83 \times 10^{-4}$ \\
Plane reorientation & $6.97 \times 10^{-12}$ & $2.20 \times 10^{-4}$ \\
\hline \hline
\end{tabular}

${ }^{a}$ Acceleration $\boldsymbol{a}$ and $\Delta \boldsymbol{V}$ are per km of amplitude in the formation. Thus, to control a $100-\mathrm{km}$ offset from the nominal costs $100 \times \Delta \boldsymbol{V}$ per year.

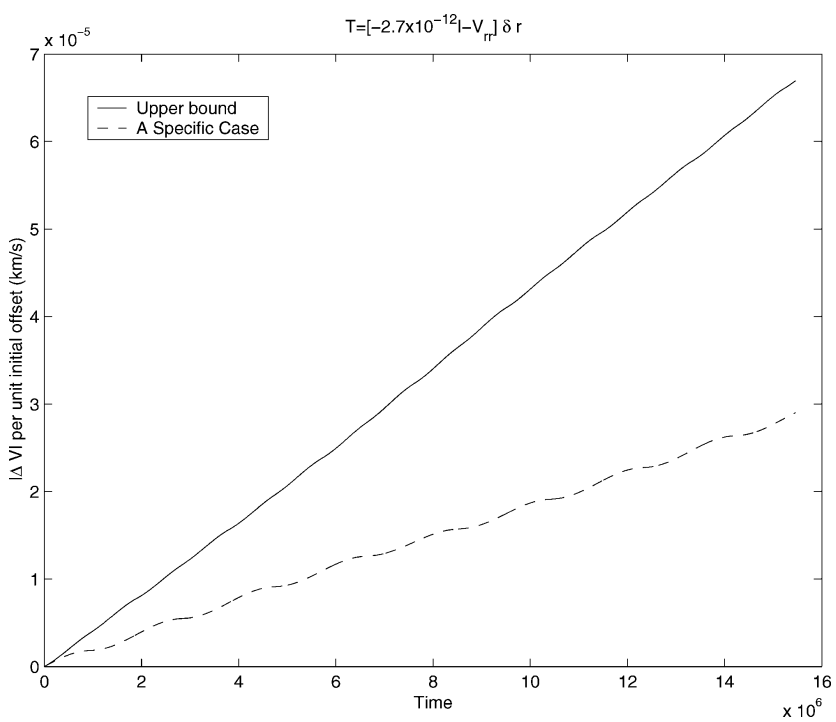

a)

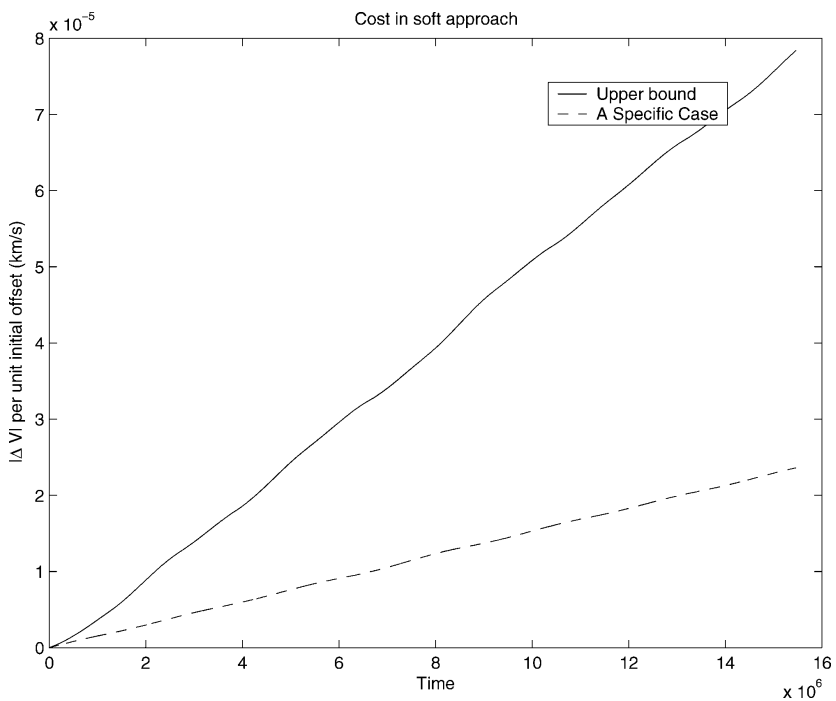

b)

Fig. 9 Upper bound on total cost per unit initial offset and the cost of a specific example with $\boldsymbol{r}_{0}=1 \mathrm{~km}$ for a) a circular trajectory generated by a position-only feedback with constant frequency over an entire period; b) a near-circular trajectory generated by a "soft" approach over an entire period; c) a circular trajectory generated by a position-and-velocity feedback over an entire period; and d) a fixed circular trajectory shown in Fig. 8. sumption are still reasonable, and we conclude that our algorithms are amenable to being implemented using low-thrust propulsion.

\section{Minimum Cost for Fixed Trajectories in the Inertial Frame}

In this section we investigate minimizing the cost to create a fixed circular trajectory in the inertial frame. Equation (60) implies that the controller is a function of system oscillation frequencies: the higher the frequency assigned, the higher the cost. Therefore, we can lower the cost by decreasing the oscillation frequencies, which are defined by the force potential matrix. As long as we keep the desired potential matrix negative definite, stability is guaranteed. However, to create a circular trajectory in the inertial frame we need to subtract the centripetal term from the desired force potential. This constraint means that we cannot design a force potential arbitrarily for the sake of stability. Consider the upper bound on cost in this case:

$$
\begin{aligned}
|\Delta \boldsymbol{V}| & =\int_{0}^{t}|\boldsymbol{a}(t)| \mathrm{d} \tau \\
& =\int_{0}^{t}\left|\left[V_{I}(t)-\tilde{\omega} \tilde{\omega}-V_{\mathrm{RR}}(t)\right] \boldsymbol{r}_{r}(t)\right| \mathrm{d} \tau
\end{aligned}
$$

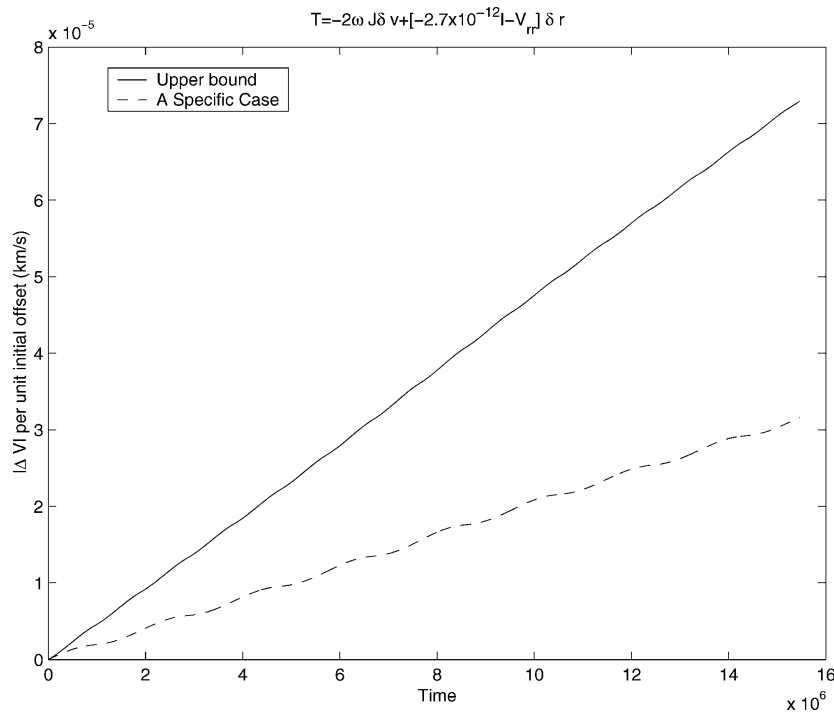

c)

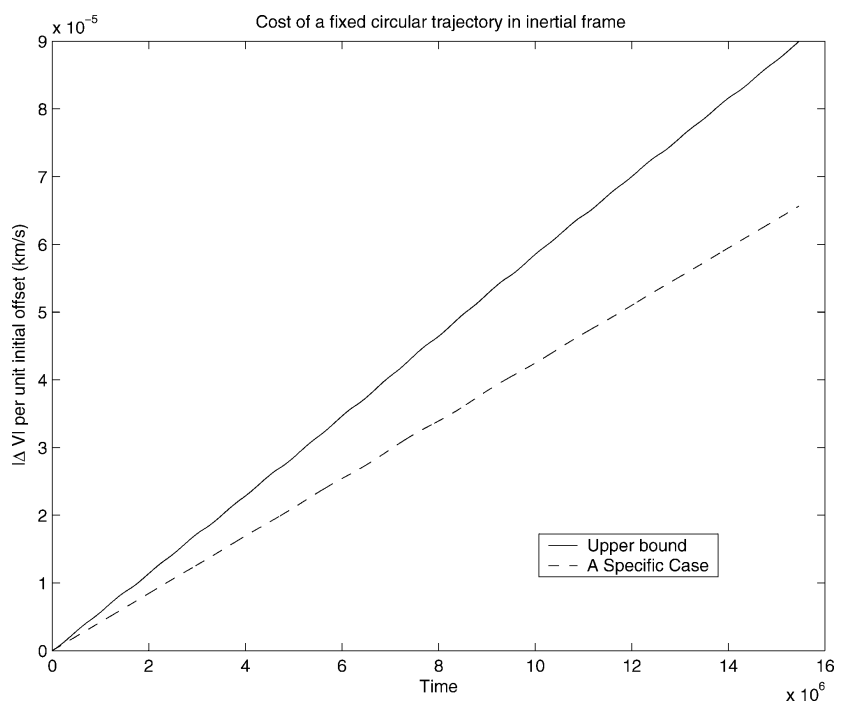

d) 


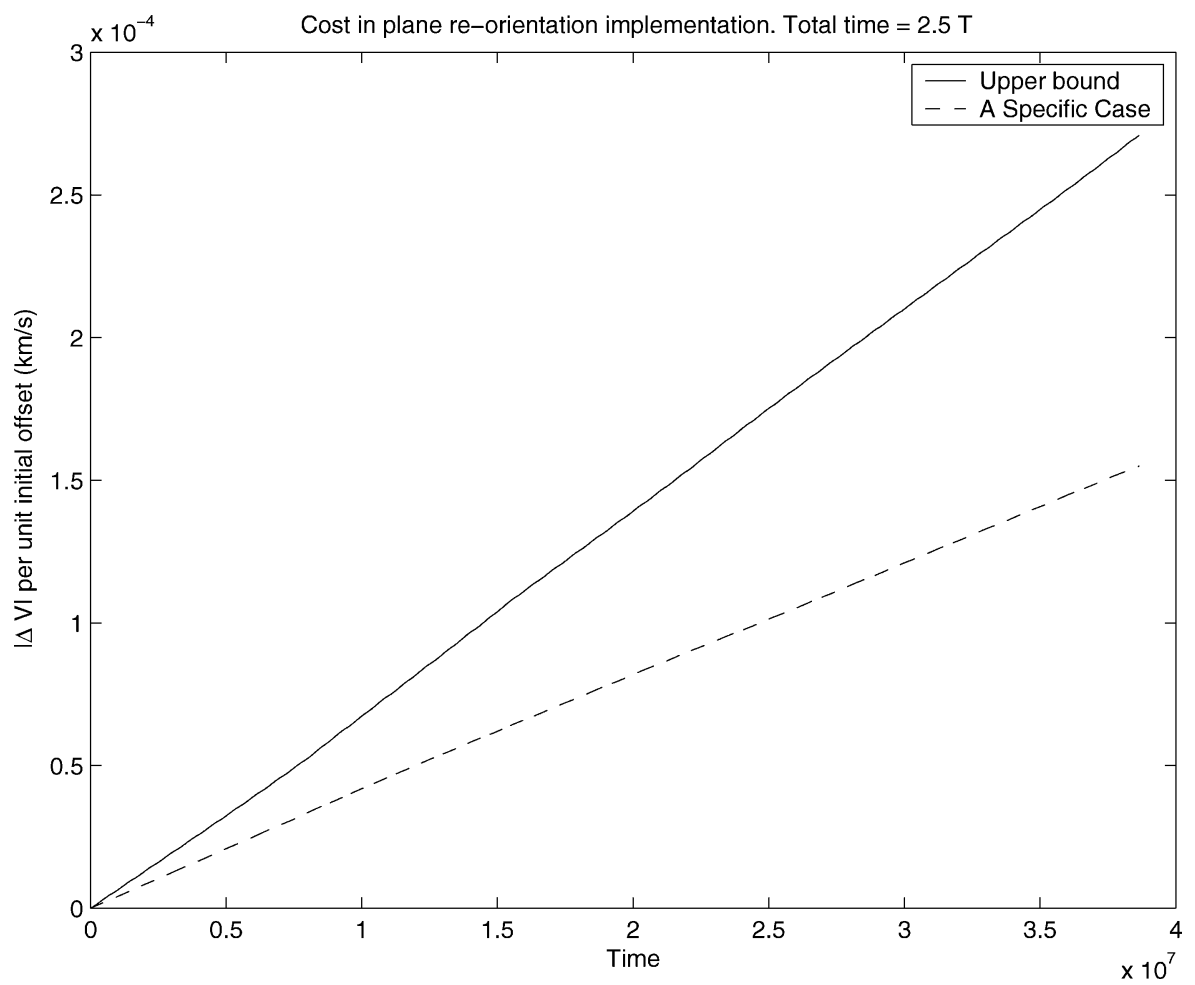

Fig. 10 Upper bound on total cost per unit initial offset in the case of plane reorientation and the cost of a specific example with $r_{0}=1 \mathrm{~km}$.

where

$$
\begin{aligned}
V_{I}(t) & =R(t) V_{i}(t) R^{T}(t) \\
& =M(t)\left[\begin{array}{ccc}
-\omega_{1}^{2}(t) & 0 & 0 \\
0 & -\omega_{2}^{2}(t) & 0 \\
0 & 0 & -\omega_{3}^{2}(t)
\end{array}\right] M^{T}(t) \\
V_{\mathrm{RR}}(t) & =\frac{3 \mu}{r_{r}^{3}(t)} \boldsymbol{r}_{r}(t) \boldsymbol{r}_{r}^{T}(t)-\frac{\mu}{r_{r}^{3}(t)} I_{3 \times 3}+\left[\begin{array}{ccc}
3 \omega_{e}^{2} & 0 & 0 \\
0 & 0 & 0 \\
0 & 0 & -\omega_{e}^{2}
\end{array}\right] \\
\tilde{\omega} \tilde{\omega} & =\omega_{e}^{2} J^{2}
\end{aligned}
$$

Let $\boldsymbol{v}_{i}$ be the $i$ th column of $M(t)$, and

$$
\begin{aligned}
\boldsymbol{r}_{r} & =c_{1} \boldsymbol{v}_{1}+c_{2} \boldsymbol{v}_{2}+c_{3} \boldsymbol{v}_{3} \\
& =M(t) \boldsymbol{c}
\end{aligned}
$$

Therefore, the integrand becomes

$$
\begin{aligned}
V_{I}-\tilde{\omega} \tilde{\omega}-V_{\mathrm{RR}}= & M\left\{\left[\begin{array}{ccc}
-\omega_{1}^{2}-2 \omega_{e}^{2} & 0 & 0 \\
0 & -\omega_{2}^{2}+\omega_{e}^{2} & 0 \\
0 & 0 & -\omega_{3}^{2}+\omega_{e}^{2}
\end{array}\right]\right. \\
& \left.+\left(\frac{-3 \mu}{r_{r}^{3}} \boldsymbol{c} c^{T}+\frac{\mu}{r_{r}^{3}} I_{3 \times 3}\right)\right\} M^{T} \\
= & M(t)[\Omega(t)+K(t)] M(t)^{T}
\end{aligned}
$$

Table 2 Comparison of acceleration and fuel bounds for generating inertially fixed circular orbits with different frequency combinations ${ }^{\mathrm{a}}$

\begin{tabular}{lcc}
\hline \hline$\left(k_{1}, k_{2}, k_{3}\right)$ & $\leq \boldsymbol{a},{ }^{\mathrm{b}} \mathrm{km} / \mathrm{s}^{2}$ & $\Delta V / \mathrm{yr},{ }^{\mathrm{b}} \mathrm{km} / \mathrm{s} / \mathrm{yr}$ \\
\hline$(1.01,1.01,1.01)$ & $8.45 \times 10^{-13}$ & $2.67 \times 10^{-5}$ \\
$(1.43,1.01,0.71)$ & $1.53 \times 10^{-12}$ & $4.83 \times 10^{-5}$ \\
$(5.03,5.03,5.03)$ & $1.88 \times 10^{-12}$ & $5.93 \times 10^{-5}$ \\
$(7.11,5.03,3.56)$ & $5.73 \times 10^{-12}$ & $1.81 \times 10^{-4}$ \\
$(10.83,10.83,10.83)$ & $6.91 \times 10^{-12}$ & $2.18 \times 10^{-4}$ \\
\hline
\end{tabular}

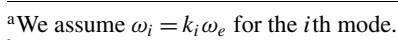

${ }^{\mathrm{b}}$ Acceleration $\boldsymbol{a}$ and $\Delta \boldsymbol{V}$ are per kilometer of amplitude in the formation. Thus, to control a $100-\mathrm{km}$ offset from the nominal costs $100 \times \Delta \boldsymbol{V}$ per year.

For simplicity, we drop $t$ in the later derivation, and Eq. (62) becomes

$$
\begin{aligned}
|\Delta \boldsymbol{V}| & =\int_{0}^{t}\left|\left[M(\Omega+K) M^{T}\right] \boldsymbol{r}_{r}\right| \mathrm{d} \tau \\
& \leq \int_{0}^{t}\left|\left(M \Omega M^{T}\right) \boldsymbol{r}_{r}\right| \mathrm{d} \tau+\int_{0}^{t}\left|\left(M K M^{T}\right) \boldsymbol{r}_{r}\right| \mathrm{d} \tau \\
& =\int_{0}^{t}\left|\sum_{i=1}^{3} \Omega_{i} c_{i} \boldsymbol{v}_{i}\right| \mathrm{d} \tau+\int_{0}^{t}|M K \boldsymbol{c}| \mathrm{d} \tau
\end{aligned}
$$

Because $M$ is orthonormal, implying that the $v_{i}$ are perpendicular to each other, we can conclude

$$
\left|\sum_{i=1}^{3} \Omega_{i} c_{i} \boldsymbol{v}_{i}\right|=\sqrt{\sum_{i=1}^{3} \Omega_{i}^{2} c_{i}^{2}}
$$

where $\Omega_{1}=-\omega_{1}^{2}-2 \omega_{e}^{2}, \Omega_{2}=-\omega_{2}^{2}+\omega_{e}^{2}, \Omega_{3}=-\omega_{3}^{2}+\omega_{e}^{2}$. By the Cauchy-Schwarz inequality,

$$
\sqrt{\sum_{i=1}^{3} \Omega_{i}^{2} c_{i}^{2}} \leq \sqrt{\sum_{i=1}^{3} \Omega_{i}^{4}} \sqrt{\sum_{i=1}^{3} c_{i}^{4}}
$$


For stability the closed-loop force potential must be negative definite, that is, $V_{I}(t)-\tilde{\omega} \tilde{\omega}<0$, implying that $\omega_{1}>\omega_{e}, \omega_{2}>\omega_{e}$, and $\omega_{3}>0$. Therefore, $|\Delta \boldsymbol{V}|$ has its minimum value when $\omega_{1} \sim \omega_{e}$, $\omega_{2} \sim \omega_{e}, \omega_{3}=\omega_{e}$. Table 2 gives a general comparison of costs for different combinations of desired oscillation frequencies. These results indicate that the above derivation for minimum cost is valid.

\section{Conclusions}

This paper develops control laws to stabilize an unstable orbit and implement formation flight designs for a reasonable fuel cost. The technique we propose defines a single feedback control law that can be distributed to all of the spacecraft in the formation. The control law is nontraditional in that it creates a new dynamical environment about the unstable orbit where the spacecraft in the formation can follow a desired path. After applying this control law, the spacecraft need to be given proper initial conditions for the entire formation to evolve in a consistent manner. The control law allows for specific planes of motion to be defined for the formation, which can either be fixed in inertial space or in an arbitrary rotating coordinate frame. Transitions of the formation between different plane orientations are easily handled by the formation simultaneously switching to a different control law, which causes the orbit plane to uniformly rotate to a new orientation. Specific examples of these formation-flight control laws in the vicinity of an unstable halo orbit in the Earth-sun system are provided and show that they can be implemented for reasonable fuel costs. Specifically, the required accelerations for maintaining our formations range from $4 \times 10^{-12}$ to $7 \times 10^{-12} \mathrm{~km} / \mathrm{s}^{2}$ (per km of formation amplitude), with the corresponding $\Delta \boldsymbol{V}$ costs ranging from $1.4 \times 10^{-4}$ to $2.2 \times 10^{-4} \mathrm{~km} / \mathrm{s} / \mathrm{yr}$ (per kilometer of formation amplitude). Using these control laws, it is possible to force a formation of spacecraft comprising a distributed interferometric observatory to lie on an image plane perpendicular to an imaging target line of sight for an arbitrary period of time, even as the entire constellation is traveling along the halo orbit. Moreover, the spacecraft in the formation will be traveling in a mutual circular orbit about the nominal trajectory, a configuration that allows an interferometric observatory to completely cover the wave-number plane in half a period, allowing for complete reconstruction of an image. The approach outlined here is a feasible way to operate a large number of satellites in an interferometric observatory using a relatively simple, and uniform, control methodology. Future issues of interest would be the robustness of this control approach to modeling and navigation errors, the effect of force perturbations, and the level of additional control needed to ensure fixed baselines between the satellites.

\section{Appendix: Proof of Lyapunov Stability}

In Eq. (18),

$$
\mathcal{V}(\delta \boldsymbol{r}, \delta \dot{\boldsymbol{r}})=-\frac{1}{2} \delta \boldsymbol{r}^{T} \tilde{V} \delta \boldsymbol{r}+\frac{1}{2} \delta \dot{\boldsymbol{r}}^{T} \delta \dot{\boldsymbol{r}}
$$

Given $\tilde{V}$ negative definite, we conclude $-\delta \boldsymbol{r}^{T} \tilde{V} \delta \boldsymbol{r} \geq 0$ and the quantity equals zero if and only if $\delta \boldsymbol{r}=0$. Then,

$$
\begin{aligned}
\mathcal{V}(\delta \boldsymbol{r}, \delta \dot{\boldsymbol{r}}) & =-\frac{1}{2} \delta \boldsymbol{r}^{T} \tilde{V} \delta \boldsymbol{r}+\frac{1}{2} \delta \dot{\boldsymbol{r}}^{T} \delta \dot{\boldsymbol{r}} \\
& =-\frac{1}{2} \delta \boldsymbol{r}^{T} \tilde{V} \delta \boldsymbol{r}+\frac{1}{2}\|\delta \dot{\boldsymbol{r}}\|^{2} \\
& \geq 0
\end{aligned}
$$

Therefore, $\mathcal{V}(\delta \boldsymbol{r}, \delta \dot{\boldsymbol{r}})=0$ if and only if $\delta \boldsymbol{r}=0$ and $\delta \dot{\boldsymbol{r}}=0$.

\section{Acknowledgments}

The work described here was funded in part by NASA's Office of Space Science and by the Interplanetary Network Technology Program by a grant from the Jet Propulsion Laboratory, California Institute of Technology, which is under contract with NASA.

\section{References}

${ }^{1}$ Barden, B. T., and Howell, K. C., "Fundamental Motions near Collinear Libration Points and Their Transitions," Journal of the Astronautical Sciences, Vol. 46, No. 4, 1998, pp. 361-378.

${ }^{2}$ Scheeres, D. J., Hsiao, F. Y., and Vinh, N. X., "Stabilizing Motion Relative to an Unstable Orbit: Applications to Spacecraft Formation Flight," Journal of Guidance, Control, and Dynamics, Vol. 26, No. 1, 2003, pp. 62-73.

${ }^{3}$ Sabol, C., Burns, R., and Mclaughlin, C. A., "Satellite Formation Flying Design and Evolution," Journal of Spacecraft and Rockets, Vol. 38, No. 2, 2001, pp. 270-278.

${ }^{4}$ Schaub, H., and Alfriend, K. T., "Impulsive Feedback Control to Establish Specific Mean Orbit Elements of Spacecraft Formations," Journal of Guidance, Control, and Dynamics, Vol. 24, No. 4, 2001, pp. 739-745.

${ }^{5}$ Hsiao, F. Y., and Scheeres, D. J., "The Dynamics of Formation Flight About a Stable Trajectory," Journal of the Astronautical Sciences, Vol. 50, No. 3, 2002, pp. 269-287.

${ }^{6}$ Hussein, I., Scheeres, D. J., and Hyland, D. C., "Interferometric Observatories in Earth Orbit," Journal of Guidance, Control, and Dynamics, Vol. 27, No. 2, 2004, pp. 297-301.

${ }^{7}$ Hsiao, F. Y., "Stabilizing and Specifying Motion Relative to Unstable Trajectories: Application to Spacecraft Formation Flight," Ph.D. Dissertation, Dept. of Aerospace Engineering, Univ. of Michigan, Ann Arbor, MI, June 2004, pp. 28-30, 64-70.

${ }^{8}$ Marchal, C., The Three-Body Problem, Elsevier, New York, 1990, p. 64.

${ }^{9}$ Howell, K. C., Barden, B. T., and Lo, M. W., "Application of Dynamical Systems Theory to Trajectory Design for a Libration Point Mission," Journal of the Astronautical Sciences, Vol. 45, No. 2, 1997, pp. 161-178.

${ }^{10}$ Nemytskii, V. V., and Stepanov, V. V., Qualitative Theory of Differential Equations, Dover, New York, 1989, p. 152.

${ }^{11}$ Bay, J. S., Fundamentals of Linear State Space Systems, McGraw-Hill, New York, 1999, p. 419.

${ }^{12}$ Liu, G. P., and Patton, R. J., Eigenstructure Assignment for Control System Design, Chichester, New York, 1998, p. 20.

${ }^{13}$ Subrahmanyan, P., and Trumper, D., "Eigenvector Assignment," Proceedings of the 1999 American Control Conference, Vol. 4, Pt. 4, American Automatic Control Council, New York, 1999, pp. 2238-2243. 